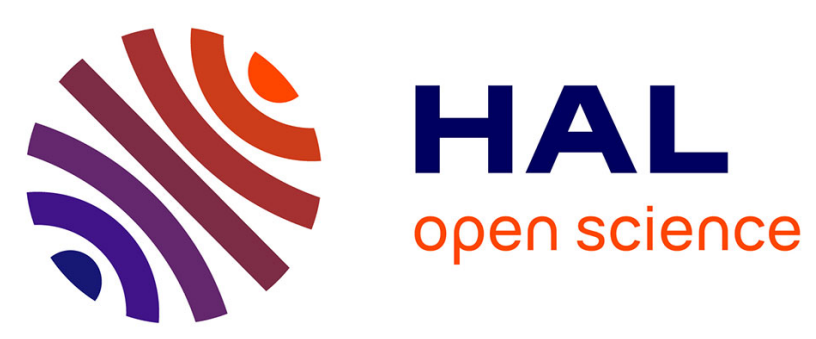

\title{
ON THE CHAPMAN-ENSKOG ASYMPTOTICS FOR A MIXTURE OF MONOATOMIC AND POLYATOMIC RAREFIED GASES
}

Céline Baranger, Marzia Bisi, Stéphane Brull, Laurent Desvillettes

\section{- To cite this version:}

Céline Baranger, Marzia Bisi, Stéphane Brull, Laurent Desvillettes. ON THE CHAPMANENSKOG ASYMPTOTICS FOR A MIXTURE OF MONOATOMIC AND POLYATOMIC RAREFIED GASES. Kinetic and Related Models , 2018. hal-01918640

\author{
HAL Id: hal-01918640 \\ https://hal.science/hal-01918640
}

Submitted on 11 Nov 2018

HAL is a multi-disciplinary open access archive for the deposit and dissemination of scientific research documents, whether they are published or not. The documents may come from teaching and research institutions in France or abroad, or from public or private research centers.
L'archive ouverte pluridisciplinaire HAL, est destinée au dépôt et à la diffusion de documents scientifiques de niveau recherche, publiés ou non, émanant des établissements d'enseignement et de recherche français ou étrangers, des laboratoires publics ou privés. 


\title{
ON THE CHAPMAN-ENSKOG ASYMPTOTICS FOR A MIXTURE OF MONOATOMIC AND POLYATOMIC RAREFIED GASES
}

\author{
CÉLINE BARANGER, MARZIA BISI, STÉPHANE BRULL, \\ AND LAURENT DESVILLETTES
}

\begin{abstract}
In this paper, we propose a formal derivation of the ChapmanEnskog asymptotics for a mixture of monoatomic and polyatomic gases. We use a direct extension of the model devised in $[8,16]$ for treating the internal energy with only one continuous parameter. This model is based on the Borgnakke-Larsen procedure [6]. We detail the dissipative terms related to the interaction between the gradients of temperature and the gradients of concentrations (Dufour and Soret effects), and present a complete explicit computation in one case when such a computation is possible, that is when all cross sections in the Boltzmann equation are constants.
\end{abstract}

\section{INTRODUCTION}

In the computations of the flow around a shuttle in the context of reentry in the upper atmosphere, it is necessary to use a kinetic description (that is, Boltzmann equations) since the Knudsen number $K n$ (defined as the mean free path of a molecule of the gas divided by a characteristic length of the shuttle) is of order 1 (or larger) at high altitude. It is also necessary to couple this kinetic description with a coherent macroscopic description used at lower altitudes where the Knudsen number becomes much smaller than 1 .

Such a coupling is well understood for one monotamic gas thanks to the establishment of the Chapman-Enskog asymptotics, which clarifies (at the formal level, cf. [2], [11], and, in a perturbative context, also at the rigorous level, cf. [24]) the relationships between the Boltzmann equation and the compressible NavierStokes(-Fourier) equations of one perfect monoatomic gas. The link between the cross section in the Boltzmann equation and the dependence of the transport coefficients (viscosity and heat conductivity) w.r.t. temperature is related to the resolution of a specific linear Boltzmann equation (cf. [15] for example), which can be solved in some specific situations, including the case of Maxwell molecules (cf. [11]). A survey on recent advances on fluid-dynamic limits of kinetic models, with both formal and rigorous proofs, may be found in [22].

It is however important to perform the Chapman-Enskog asymptotics in situations much more complicated than the ones in which is considered only one single monoatomic gas. Indeed, the main chemical species found in the upper atmosphere of the earth are the molecular oxygen $\left(\mathrm{O}_{2}\right)$ and the molecular nitrogen $\left(N_{2}\right)$, which are both diatomic. Moreover, due to the chemical (dissociation/recombination) reactions taking place in the heated air surrounding a shuttle, one should also (at least) take into account the atomic oxygen $O$, the atomic nitrogen $N$ (both 
are obviously monoatomic) and the diatomic nitrogen monoxide $N O$. As a consequence, it is important to be able to treat mixtures of several monoatomic and polyatomic gases with different masses (note that it is possible to approximate the masses of $\mathrm{N}_{2}, \mathrm{O}_{2}$ and $\mathrm{NO}$ by a common value, but this cannot be generalized if one takes into account the (atomic) argon $A r$, whose concentration in the upper atmosphere is not unsignificant). Other interesting physical applications involving polyatomic gases are discussed in the monographs [34], where transport phenomena in multi-component plasmas are examined, and [29], where the authors investigate conditions of a strongly vibrational and chemical non-equilibrium state, developing methods of kinetic theory in the approximation of the state-to-state kinetics.

Our goal is to present in detail the Chapman-Enskog asymptotics in a model as simple as possible fulfilling the assumptions described above (that is, taking into account a mixture of several monoatomic and polyatomic gases with different masses), and which enables to recover at the macroscopic level a set of compressible Navier-Stokes equations for perfect gases with general energy laws. The model proposed in $[8,16]$ almost fulfills those assumptions. It uses as unknowns the number densities in the phase space $f^{(i)}(t, x, v, I)$ of particles of the $i$-th species which at time $t$ and point $x$ move with velocity $v$ and have a one-dimensional internal energy parameter $I>0$. The choice of one parameter in the model enables to get quite general energy equations, but unfortunately not the energy equation of monoatomic gases (which can be recovered only as a limit of the model). In order to integrate the possibility of having mixtures of monoatomic and polyatomic species, we introduce therefore in the model of [16] collision kernels for monoatomicdiatomic collisions (these kernels are described in section 2). For some applications of such models we refer to [25], [32], [20]. In particular in [25], the authors highlight different types of shock profiles which are specific to the polyatomic setting by using the model given in [1], [10], [26]. In [17], a numerical model for polyatomic gases using the reduced distribution technique is derived.

In order to test the compatibility of numerical (usually DSMC) codes used at the kinetic level with fluid mechanics codes used at the macroscopic level, it is useful to have one example in which the transport coefficients can be explicitly derived from the cross sections used in the Boltzmann equation. We provide in this paper such an explicit computation (that is, when the cross sections are constants). This computation can be seen as an extension of classical computations of transport coefficients for monoatomic gases with a cross section of Maxwell molecules type (cf. [11]).

We notice that in [18], [21], [19], [33] the authors describe the internal energy variable with a discrete parameter. In [18], [21], [19], a Chapman-Enskog expansion is performed starting from the Boltzmann collision operator given in [33]. This way of modelling has been adopted in [23], [4], where kinetic equations of Boltzmann or BGK-type are built up for mixtures of gases undergoing also a bimolecular reversible chemical reaction. In [4] the hydrodynamic limit of the BGK model for a fast reactive mixture of monatomic gases is derived, at both Euler and Navier-Stokes levels, by a Chapman-Enskog procedure in terms of the relevant hydrodynamic variables. This BGK model has been recently generalized in [3] to a mixture of polyatomic gases (inert or reacting), each one having a set of discrete energy levels; the relevant asymptotic limit is available only for a single gas, and its comparison with phenomenological results obtained in the frame of Extended 
Thermodynamics seems to be promising [5]. Suitable fluid-dynamic closures for a single polyatomic gas have been achieved in the case of a continuous internal energy [30], and the state of the art on the matter may be found in the book [31]. However, for the reasons explained above, in view of practical applications, it is important to provide a complete Navier-Stokes description for a mixture involving monoatomic and polyatomic species, and this is the aim of our work.

The paper is organised as follows. In section 2, the kinetic model for mixtures of monoatomic and polyatomic gases is introduced, Boltzmann kernels are written down together with the corresponding linear operators, and conservations laws associated to the kernels are recalled. In section 3, the asymptotic expansion is performed, and the various transport terms appearing in the Navier Stokes system are described and linked to the cross sections of the Boltzmann kernels. Then, section 4 is devoted to the complete treatment of the case when all cross sections are constant: in this case all transport terms can be made explicit, obtaining thus Navier-Stokes equations consistent with the physical expectations (see [28], [21]). Some basic integrals widely used in the procedure are finally listed in a short Appendix A, and some steps of the computations needed in Section 4 are detailed in Appendix B.

\section{BOLTZMANN KERNELS FOR A MIXTURE OF RAREFIED MONOATOMIC AND POLYATOMIC GASES}

In this section, we present a direct extension of the model devised in [16] to the case of a mixture of monoatomic and polyatomic gases.

2.1. General definitions. We consider a mixture of $A$ monoatomic gases and $B$ polyatomic gases. The distribution function (at time $t$, point $x$ and velocity $v$ ) of each monoatomic species $i \in\{1, \ldots, A\}$ is denoted by $f^{(i)}(t, x, v)$, where $(t, x, v) \in \mathbb{R}_{+} \times \mathbb{R}^{3} \times \mathbb{R}^{3}$. Then, we introduce for the polyatomic species $i \in\{A+$ $1, \ldots, A+B\}$ a unique continuous energy variable $I \in \mathbb{R}_{+}$, collecting rotational and vibrational energies. Therefore the polyatomic species are represented by the quantity $f^{(i)}(t, x, v, I)$, where $(t, x, v, I) \in \mathbb{R}_{+} \times \mathbb{R}^{3} \times \mathbb{R}^{3} \times \mathbb{R}_{+}$. Following [8] and [16], we introduce (for each poyatomic species $i=A+1, \ldots, A+B$ ) a function $\varphi_{i}(I)>0$, which is a parameter of the model. This function is related to the energy law obtained at the macroscopic level for the considered species $i$ (cf. [14]), for example $\varphi_{i}(I)=1$ for the energy law of diatomic gases $e=\frac{5}{2} T$ ( $e$ being the macroscopic internal energy by unit of mass, and $T$ being the temperature, computed in a unit such that the constant of perfect gases is 1 ).

In the following, the quantity $f^{(i)} \varphi_{i}$ represents the classical distribution function (see Remark 1).

Note that the discrete internal energy levels obtained from quantum mechanics enable a much more detailed description of the rotational and vibrational states of a polyatomic molecule than the crude Borgnakke-Larsen procedure used in this paper.

The interest of using this procedure (and of considering one single continuous internal energy parameter) resides in the very simple way in which it can then be implemented in (already existing) DSMC numerical codes used in an engineering context. 
Finally we define the mass $m_{i}$ of a molecule of species $i$, and recall the definition of macroscopic quantities:

The (macroscopic) mass of monoatomic species $i \in\{1, \ldots, A\}$ (at time $t$ and point $x)$ :

$$
\rho^{(i)}=m_{i} n^{(i)}(t, x):=\int_{\mathbb{R}^{3}} f^{(i)}(t, x, v) m_{i} d v .
$$

The (macroscopic) mass of polyatomic species $i \in\{A+1, \ldots, A+B\}$ (at time $t$ and point $x)$ :

$$
\rho^{(i)}=m_{i} n^{(i)}(t, x):=\int_{\mathbb{R}^{3}} \int_{0}^{\infty} f^{(i)}(t, x, v, I) m_{i} \varphi_{i}(I) d I d v .
$$

The momentum of monoatomic species $i \in\{1, \ldots, A\}$ (at time $t$ and point $x$ ):

$$
m_{i} n^{(i)}(t, x) u^{(i)}(t, x):=\int_{\mathbb{R}^{3}} f^{(i)}(t, x, v) m_{i} v d v .
$$

The momentum of polyatomic species $i \in\{A+1, \ldots, A+B\}$ (at time $t$ and point $x)$ :

$$
m_{i} n^{(i)}(t, x) u^{(i)}(t, x):=\int_{\mathbb{R}^{3}} \int_{0}^{\infty} f^{(i)}(t, x, v, I) m_{i} v \varphi_{i}(I) d I d v .
$$

The (macroscopic, internal) energy of monoatomic species $i \in\{1, \ldots, A\}$ (at time $t$ and point $x$ ):

$$
m_{i} n^{(i)}(t, x) e^{(i)}(t, x):=\int_{\mathbb{R}^{3}} f^{(i)}(t, x, v) m_{i} \frac{\left|v-u^{(i)}(t, x)\right|^{2}}{2} d v .
$$

The (macroscopic, internal) energy of polyatomic species $i \in\{A+1, \ldots, A+B\}$ (at time $t$ and point $x$ ):

$m_{i} n^{(i)}(t, x) e^{(i)}(t, x):=\int_{\mathbb{R}^{3}} \int_{0}^{\infty} f^{(i)}(t, x, v, I)\left(m_{i} \frac{\left|v-u^{(i)}(t, x)\right|^{2}}{2}+I\right) \varphi_{i}(I) d I d v$.

Since in this work we do not study chemically reactive collisions, we do not introduce the formation energies $e_{i}^{0}$. These energies should of course be introduced if chemically reactive collisions were considered. They would enable to recover the form of the Navier-Stokes systems which are used in combustion theory.

2.2. Collision operators. In this subsection, we define the collision operators enabling to treat the collisions between the various types of gases (monoatomic and polyatomic).

2.2.1. Collision operator for monoatomic species. We write here the usual Boltzmann kernel, for collisions between species $i$ and $j(i, j \in\{1, \ldots, A\})$.

We define (for $f:=f(v) \geq 0, g:=g(v) \geq 0$ ):

$Q_{i j}(f, g)(v)=\int_{\mathbb{R}^{3}} \int_{S^{2}}\left\{f\left(v^{\prime}\right) g\left(v_{*}^{\prime}\right)-f(v) g\left(v_{*}\right)\right\} B_{i j}\left(\left|v-v_{*}\right|, \frac{v-v_{*}}{\left|v-v_{*}\right|} \cdot \sigma\right) d \sigma d v_{*}$,

with

$$
v^{\prime}=\frac{m_{i} v+m_{j} v_{*}}{m_{i}+m_{j}}+\frac{m_{j}}{m_{i}+m_{j}}\left|v-v_{*}\right| \sigma
$$




$$
v_{*}^{\prime}=\frac{m_{i} v+m_{j} v_{*}}{m_{i}+m_{j}}-\frac{m_{i}}{m_{i}+m_{j}}\left|v-v_{*}\right| \sigma .
$$

The cross section $B_{i j}$ satisfies the symmetry constraint $B_{i j}=B_{j i}$. As a consequence, the operator satisfies the following weak formulation: For $\psi_{i}:=\psi_{i}(v)$, $\psi_{j}:=\psi_{j}(v)$,

$$
\begin{gathered}
\int_{\mathbb{R}^{3}} Q_{i j}(f, g)(v) \psi_{i}(v) d v+\int_{\mathbb{R}^{3}} Q_{j i}(g, f)(v) \psi_{j}(v) d v \\
=-\frac{1}{2} \int_{\mathbb{R}^{3}} \int_{\mathbb{R}^{3}} \int_{S^{2}}\left\{f\left(v^{\prime}\right) g\left(v_{*}^{\prime}\right)-f(v) g\left(v_{*}\right)\right\} \times\left(\psi_{i}\left(v^{\prime}\right)+\psi_{j}\left(v_{*}^{\prime}\right)-\psi_{i}(v)-\psi_{j}\left(v_{*}\right)\right) \\
\times B_{i j}\left(\left|v-v_{*}\right|, \frac{v-v_{*}}{\left|v-v_{*}\right|} \cdot \sigma\right) d \sigma d v_{*} d v .
\end{gathered}
$$

This weak formulation implies the conservation of momentum and kinetic energy:

$$
\int_{\mathbb{R}^{3}} Q_{i j}(f, g)(v)\left(\begin{array}{c}
m_{i} v \\
m_{i} \frac{|v|^{2}}{2}
\end{array}\right) d v+\int_{\mathbb{R}^{3}} Q_{j i}(g, f)(v)\left(\begin{array}{c}
m_{j} v \\
m_{j} \frac{|v|^{2}}{2}
\end{array}\right) d v=\left(\begin{array}{c}
0 \\
0
\end{array}\right),
$$

together with the entropy inequality:

$$
\int_{\mathbb{R}^{3}} Q_{i j}(f, g)(v) \ln f(v) d v+\int_{\mathbb{R}^{3}} Q_{j i}(g, f)(v) \ln g(v) d v \leq 0 .
$$

2.2.2. Collision operators between monoatomic and polyatomic molecules. We write here the asymmetric operator enabling to treat the collisions between a polyatomic molecule (of mass $m_{i}$, with $i \in\{A+1, \ldots, A+B\}$ ), and a monoatomic one (of mass $m_{j}$, with $\left.j \in\{1, \ldots, A\}\right)$. This operator is inspired from the operators presented in $[8],[14],[16]$.

We define (for $f:=f(v, I)$ and $g:=g(v))$ :

$$
\begin{aligned}
& Q_{i j}(f, g)(v, I)=\int_{\mathbb{R}^{3}} \int_{S^{2}} \int_{0}^{1}\left\{f\left(v^{\prime}, I^{\prime}\right) g\left(v_{*}^{\prime}\right)-f(v, I) g\left(v_{*}\right)\right\} \\
& \times B_{i j}\left(\sqrt{E}, R^{1 / 2}\left|v-v_{*}\right|, \frac{v-v_{*}}{\left|v-v_{*}\right|} \cdot \sigma\right) R^{1 / 2} \varphi_{i}(I)^{-1} d R d \sigma d v_{*},
\end{aligned}
$$

with

$$
\begin{gathered}
v^{\prime}=\frac{m_{i} v+m_{j} v_{*}}{m_{i}+m_{j}}+\frac{m_{j}}{m_{i}+m_{j}} \sqrt{\frac{2 R E}{\mu_{i j}}} \sigma, \\
v_{*}^{\prime}=\frac{m_{i} v+m_{j} v_{*}}{m_{i}+m_{j}}-\frac{m_{i}}{m_{i}+m_{j}} \sqrt{\frac{2 R E}{\mu_{i j}}} \sigma, \\
I^{\prime}=(1-R) E,
\end{gathered}
$$

where $\mu_{i j}=\frac{m_{i} m_{j}}{m_{i}+m_{j}}$ is the reduced mass, $E=\frac{1}{2} \mu_{i j}\left|v-v_{*}\right|^{2}+I$ is the total energy of the two molecules in the center of mass reference frame, and the parameter $R$ lies in $[0,1]$.

We also define the symmetric operator (with the same cross section)

$$
Q_{j i}(g, f)(v)=\int_{\mathbb{R}^{3}} \int_{0}^{\infty} \int_{S^{2}} \int_{0}^{1}\left\{g\left(v^{\prime}\right) f\left(v_{*}^{\prime}, I_{*}^{\prime}\right)-g(v) f\left(v_{*}, I_{*}\right)\right\}
$$


with

$$
\times B_{i j}\left(\sqrt{E}, R^{1 / 2}\left|v-v_{*}\right|, \frac{v-v_{*}}{\left|v-v_{*}\right|} \cdot \sigma\right) R^{1 / 2} d R d \sigma d v_{*} d I_{*}
$$

$$
\begin{gathered}
v^{\prime}=\frac{m_{j} v+m_{i} v_{*}}{m_{i}+m_{j}}+\frac{m_{i}}{m_{i}+m_{j}} \sqrt{\frac{2 R E}{\mu_{i j}}} \sigma, \\
v_{*}^{\prime}=\frac{m_{j} v+m_{i} v_{*}}{m_{i}+m_{j}}-\frac{m_{j}}{m_{i}+m_{j}} \sqrt{\frac{2 R E}{\mu_{i j}}} \sigma, \\
I_{*}^{\prime}=(1-R) E,
\end{gathered}
$$

where $\mu_{i j}=\frac{m_{i} m_{j}}{m_{i}+m_{j}}$ and $E=\frac{1}{2} \mu_{i j}\left|v-v_{*}\right|^{2}+I_{*}$.

These operators satisfy the following weak formulation (note that by symmetry, the same cross section $B_{i j}$ appears in $Q_{i j}$ and $\left.Q_{j i}\right)$ : for $\psi_{i}:=\psi_{i}(v, I) \geq 0, \psi_{j}:=$ $\psi_{j}(v) \geq 0$

$$
\begin{gathered}
\int_{\mathbb{R}^{3}} \int_{0}^{\infty} Q_{i j}(f, g)(v, I) \psi_{i}(v, I) \varphi_{i}(I) d v d I+\int_{\mathbb{R}^{3}} Q_{j i}(g, f)(v) \psi_{j}(v) d v \\
=-\frac{1}{2} \int_{\mathbb{R}^{3}} \int_{\mathbb{R}^{3}} \int_{0}^{\infty} \int_{S^{2}} \int_{0}^{1}\left\{f\left(v^{\prime}, I^{\prime}\right) g\left(v_{*}^{\prime}\right)-f(v, I) g\left(v_{*}\right)\right\} \\
\times\left(\psi_{i}\left(v^{\prime}, I^{\prime}\right)+\psi_{j}\left(v_{*}^{\prime}\right)-\psi_{i}(v, I)-\psi_{j}\left(v_{*}\right)\right) B_{i j}\left(\sqrt{E}, R^{1 / 2}\left|v-v_{*}\right|, \frac{v-v_{*}}{\left|v-v_{*}\right|} \cdot \sigma\right) R^{1 / 2} d R d \sigma d v_{*} d I d v .
\end{gathered}
$$

The weak formulation implies the conservation of momentum and total energy:

$$
\int_{\mathbb{R}^{3}} \int_{0}^{\infty} Q_{i j}(f, g)(v, I) \varphi_{i}(I)\left(\begin{array}{c}
m_{i} v \\
m_{i} \frac{|v|^{2}}{2}+I
\end{array}\right) d I d v+\int_{\mathbb{R}^{3}} Q_{j i}(g, f)(v)\left(\begin{array}{c}
m_{j} v \\
m_{j} \frac{|v|^{2}}{2}
\end{array}\right) d v=\left(\begin{array}{c}
0 \\
0
\end{array}\right),
$$

together with the entropy inequality:

$$
\int_{\mathbb{R}^{3}} \int_{0}^{\infty} Q_{i j}(f, g)(v, I) \ln f(v, I) \varphi_{i}(I) d I d v+\int_{\mathbb{R}^{3}} Q_{j i}(g, f)(v) \ln g(v) d v \leq 0 .
$$

2.2.3. Collision operators for polyatomic molecules. We finally present the operator enabling to treat the collisions between two polyatomic molecules of respective mass $m_{i}$ and $m_{j}(i, j \in\{A+1, \ldots, A+B\})$. This operator is extracted from [16] (in the case when no chemical reactions are considered).

We define $($ for $f:=f(v, I) \geq 0, g:=g(v, I) \geq 0)$ :

$$
\begin{aligned}
& Q_{i j}(f, g)(v, I)=\int_{\mathbb{R}^{3}} \int_{0}^{\infty} \int_{S^{2}} \int_{0}^{1} \int_{0}^{1}\left\{f\left(v^{\prime}, I^{\prime}\right) g\left(v_{*}^{\prime}, I_{*}^{\prime}\right)-f(v, I) g\left(v_{*}, I_{*}\right)\right\} \\
& \times B_{i j}\left(\sqrt{E}, R^{1 / 2}\left|v-v_{*}\right|, \frac{v-v_{*}}{\left|v-v_{*}\right|} \cdot \sigma\right)(1-R) R^{1 / 2} \varphi_{i}(I)^{-1} d r d R d \sigma d I_{*} d v_{*},
\end{aligned}
$$

with

$$
\begin{aligned}
& v^{\prime}=\frac{m_{i} v+m_{j} v_{*}}{m_{i}+m_{j}}+\frac{m_{j}}{m_{i}+m_{j}} \sqrt{\frac{2 R E}{\mu_{i j}}} \sigma, \\
& v_{*}^{\prime}=\frac{m_{i} v+m_{j} v_{*}}{m_{i}+m_{j}}-\frac{m_{i}}{m_{i}+m_{j}} \sqrt{\frac{2 R E}{\mu_{i j}}} \sigma,
\end{aligned}
$$




$$
I^{\prime}=r(1-R) E, \quad I_{*}^{\prime}=(1-r)(1-R) E,
$$

where $\mu_{i j}=\frac{m_{i} m_{j}}{m_{i}+m_{j}}$ is the reduced mass, $E=\frac{1}{2} \mu_{i j}\left|v-v_{*}\right|^{2}+I+I_{*}$ is the total energy of the two molecules in the center of mass reference frame, and $r, R$ lie in $[0,1]$.

Remark 1. The collision operator defined in (11) can be rewritten as

$$
\begin{gathered}
Q_{i j}\left(f_{i}, f_{j}\right)(v, I)=\int_{\mathbb{R}^{3}} \int_{0}^{\infty} \int_{S^{2}} \int_{0}^{1} \int_{0}^{1}\left\{\frac{\varphi_{i}(I) \varphi_{j}\left(I_{*}\right)}{\varphi_{i}\left(I^{\prime}\right) \varphi_{j}\left(I_{*}^{\prime}\right)} \varphi_{i}\left(I^{\prime}\right) f_{i}\left(v^{\prime}, I^{\prime}\right) \varphi_{j}\left(I_{*}^{\prime}\right) f_{j}\left(v_{*}^{\prime}, I_{*}^{\prime}\right)\right. \\
\left.-\varphi_{i}(I) f_{i}(v, I) \varphi_{j}\left(I_{*}\right) f_{j}\left(v_{*}, I_{*}\right)\right\} \\
\times \frac{B_{i j}\left(\sqrt{E}, R^{1 / 2}\left|v-v_{*}\right|, \frac{v-v_{*}}{\left|v-v_{*}\right|} \cdot \sigma\right)}{\varphi_{i}(I) \varphi_{j}\left(I_{*}\right)}(1-R) R^{1 / 2} \varphi_{i}(I)^{-1} d r d R d \sigma d I_{*} d v_{*} .
\end{gathered}
$$

In this expression, $\varphi_{i} f_{i}$ represents the effective distribution function and $\frac{B_{i j}}{\varphi_{i}(I) \varphi_{j}\left(I_{*}\right)}$ corresponds to the effective collision cross section. Note that using this description of $Q_{i j}$, we recover the general shape of the collision operators presented for example in [8], [18], [21].

Note also that the microreversibility property of $B_{i j}$ (cf. [16]) is consistent with the one appearing in [33].

Using the symmetry constraints $B_{i j}=B_{j i}$, one can show that these operators satisfy the following weak formulation: for $\psi_{i}:=\psi_{i}(v, I), \psi_{j}:=\psi_{j}(v, I)$,

$$
\begin{aligned}
\int_{\mathbb{R}^{3}} \int_{0}^{\infty} Q_{i j}(f, g)(v, I) \psi_{i}(v, I) \varphi_{i}(I) d v d I+\int_{\mathbb{R}^{3}} \int_{0}^{\infty} Q_{j i}(g, f)(v) \psi_{j}(v, I) \varphi_{j}(I) d v d I \\
=-\frac{1}{2} \int_{\mathbb{R}^{3}} \int_{0}^{\infty} \int_{\mathbb{R}^{3}} \int_{0}^{\infty} \int_{S^{2}} \int_{0}^{1} \int_{0}^{1}\left\{f\left(v^{\prime}, I^{\prime}\right) g\left(v_{*}^{\prime}, I_{*}^{\prime}\right)-f(v, I) g\left(v_{*}, I_{*}\right)\right\} \\
\times\left(\psi_{i}\left(v^{\prime}, I^{\prime}\right)+\psi_{j}\left(v_{*}^{\prime}, I_{*}^{\prime}\right)-\psi_{i}(v, I)-\psi_{j}\left(v_{*}, I_{*}\right)\right) \\
\times B_{i j}\left(\sqrt{E}, R^{1 / 2}\left|v-v_{*}\right|, \frac{v-v_{*}}{\left|v-v_{*}\right|} \cdot \sigma\right)(1-R) R^{1 / 2} d r d R d \omega d I_{*} d v_{*} d I d v .
\end{aligned}
$$

This weak formulation implies the conservation of momentum and total energy:

$$
\begin{gathered}
\int_{\mathbb{R}^{3}} \int_{0}^{\infty} Q_{i j}(f, g)(v, I)\left(\begin{array}{c}
m_{i} v \\
m_{i} \frac{|v|^{2}}{2}+I
\end{array}\right) \varphi_{i}(I) d I d v \\
+\int_{\mathbb{R}^{3}} \int_{0}^{\infty} Q_{j i}(g, f)(v, I)\left(\begin{array}{c}
m_{j} v \\
m_{j} \frac{|v|^{2}}{2}+I
\end{array}\right) \varphi_{j}(I) d I d v=\left(\begin{array}{l}
0 \\
0
\end{array}\right),
\end{gathered}
$$

together with the entropy inequality:

$$
\begin{gathered}
\int_{\mathbb{R}^{3}} \int_{0}^{\infty} Q_{i j}(f, g)(v, I) \ln f(v, I) \varphi_{i}(I) d I d v \\
+\int_{\mathbb{R}^{3}} \int_{0}^{\infty} Q_{j i}(g, f)(v, I) \ln g(v, I) \varphi_{j}(I) d I d v \leq 0 .
\end{gathered}
$$


2.3. Linearized operators. We now introduce the Maxwellian distributions

$$
M^{(i)}:=\frac{n^{(i)}}{\left(2 \pi T / m_{i}\right)^{3 / 2} q_{i}(T)} \exp \left(-\frac{m_{i}|v-u|^{2}+2 r_{i} I}{2 T}\right),
$$

with $r_{i}=0$ for $i=1, \ldots, A$ and $r_{i}=1$ for $i=A+1, \ldots, A+B$.

In the formula above, $q_{i}(T)=1$ for $i=1, \ldots, A$ and

$$
q_{i}(T):=\int_{0}^{\infty} \varphi_{i}(I) e^{-I / T} d I
$$

for $i=A+1, \ldots, A+B$. We refer to [14] and [16] for those formulas in the case when $r_{i}=1$. In the framework of [21], [19], this term is considered as an internal energy partition of species $i$.

For any family of functions $g^{(i)}:=g^{(i)}(v, I)$ with $i=A+1, \ldots, A+B$, one can write

$$
\begin{gathered}
{\left[\left[M^{(i)}\right]^{-1} Q_{i j}\left(M^{(i)}, M^{(j)} g^{(j)}\right)+\left[M^{(i)}\right]^{-1} Q_{i j}\left(M^{(i)} g^{(i)}, M^{(j)}\right)\right](\sqrt{T} V+u, J T)} \\
=n^{(j)} K_{i j}\left((v, I) \mapsto g^{(i)}(v \sqrt{T}+u, I T),(v, I) \mapsto g^{(j)}(v \sqrt{T}+u, I T)\right)(V, J),
\end{gathered}
$$

where $K_{i j}$ is defined below. Formulas very close to (18) can be written down when at least one of the molecules is monoatomic (the only difference being that the dependence w.r.t. the second variable of $g^{(i)}$ and/or $g^{(j)}$ does not appear).

We define now the global linearized operator (around a centered reduced Maxwellian, and with a rescaled cross section) as

$$
\mathcal{K}:\left(\begin{array}{c}
h^{(1)} \\
\cdot \\
\dot{h^{(A+B)}}
\end{array}\right) \mapsto\left(\begin{array}{c}
\sum_{j} n^{(j)} K_{1 j}\left(h^{(1)}, h^{(j)}\right) \\
\cdot \\
\sum_{j} n^{(j)} K_{A+B j}\left(h^{(A+B)}, h^{(j)}\right)
\end{array}\right) .
$$

This operator will play an important role in the study of the Chapman-Enskog asymptotics described in next section, and components $K_{i j}$ vary according to what type of interactions we are dealing with (among monatomic molecules, or polyatomic molecules, or pairs of a monatomic and a polyatomic molecule).

Thanks to the entropy inequalities satisfied by the $Q_{i j}$ (such as (15)), it is possible to show that $\mathcal{K}$ is symmetric and semi-definite negative (so that often, one considers $-\mathcal{K})$, w.r.t to a scalar product defined below.

Note also that $\mathcal{K}$ is Galilean-invariant (isotropic w.r.t. the velocity variable) in the following sense: for all isometric transformation $\mathcal{R}$ in $O(3, \mathbb{R}$ ), one has (denoting by $o$ the composition w.r.t the velocity variable only),

$$
\mathcal{K}\left(h^{(1)} o \mathcal{R}, \ldots, h^{(A+B)} o \mathcal{R}\right)(v, I)=\mathcal{K}\left(h^{(1)}, \ldots, h^{(A+B)}\right)(\mathcal{R} v, I) .
$$

We refer for example to [15] for a complete proof of these properties in the case of one single monoatomic gas.

We begin the description of the $K_{i j}$ in the monoatomic-monoatomic case. For $i=1, \ldots, A, j=1, \ldots, A$,

$$
K_{i j}\left(h^{(i)}, h^{(j)}\right)(v)=\int_{\mathbb{R}^{3}} \int_{S^{2}} \frac{e^{-\frac{m_{j}}{2}\left|v_{*}\right|^{2}}}{\left(2 \pi / m_{j}\right)^{3 / 2}}
$$




$$
\times\left[h^{(j)}\left(v_{*}^{\prime}\right)+h^{(i)}\left(v^{\prime}\right)-h^{(j)}\left(v_{*}\right)-h^{(i)}(v)\right] B_{i j}\left(\sqrt{T}\left|v-v_{*}\right|, \frac{v-v_{*}}{\left|v-v_{*}\right|} \cdot \sigma\right) d \sigma d v_{*}
$$

where $v^{\prime}, v_{*}^{\prime}$ are defined in (2), (3).

We then turn to the monoatomic-polyatomic case. For $i=1, \ldots, A, j=A+$ $1, \ldots, A+B$

$$
\begin{gathered}
K_{i j}\left(h^{(i)}, h^{(j)}\right)(v)=\int_{\mathbb{R}^{3}} \int_{0}^{\infty} \int_{S^{2}} \int_{0}^{1} \frac{e^{-\frac{m_{j}}{2}\left|v_{*}\right|^{2}-I_{*}}}{\left(2 \pi / m_{j}\right)^{3 / 2}} \\
\times\left[h^{(j)}\left(v_{*}^{\prime},(1-R) E\right)+h^{(i)}\left(v^{\prime}\right)-h^{(j)}\left(v_{*}, I_{*}\right)-h^{(i)}(v)\right] \\
\times \frac{T}{q_{j}(T)} B_{i j}\left(\sqrt{T} \sqrt{E}, \sqrt{T} R^{1 / 2}\left|v-v_{*}\right|, \frac{v-v_{*}}{\left|v-v_{*}\right|} \cdot \sigma\right) R^{1 / 2} d R d \sigma d I_{*} d v_{*},
\end{gathered}
$$

with $E=\frac{1}{2} \mu_{i j}\left|v-v_{*}\right|^{2}+I_{*}$ and $v^{\prime}, v_{*}^{\prime}$ defined in (5), (6).

Symmetrically, we write down the polyatomic-monoatomic case. For $i=A+$ $1, \ldots, A+B, j=1, \ldots, A$,

$$
\begin{gathered}
K_{i j}\left(h^{(i)}, h^{(j)}\right)(v, I)=\int_{\mathbb{R}^{3}} \int_{S^{2}} \int_{0}^{1} \frac{e^{-\frac{m_{j}}{2}\left|v_{*}\right|^{2}}}{\left(2 \pi / m_{j}\right)^{3 / 2}} \\
\times\left[h^{(j)}\left(v_{*}^{\prime}\right)+h^{(i)}\left(v^{\prime},(1-R) E\right)-h^{(j)}\left(v_{*}\right)-h^{(i)}(v, I)\right] \\
\times B_{i j}\left(\sqrt{T} \sqrt{E}, \sqrt{T} R^{1 / 2}\left|v-v_{*}\right|, \frac{v-v_{*}}{\left|v-v_{*}\right|} \cdot \sigma\right) R^{1 / 2} \varphi_{i}(I)^{-1} d R d \sigma d v_{*},
\end{gathered}
$$

with $E=\frac{1}{2} \mu_{i j}\left|v-v_{*}\right|^{2}+I$ and $v^{\prime}, v_{*}^{\prime}$ are defined in (8), (9).

Finally, we consider the polyatomic-polyatomic case. For $i=A+1, \ldots, A+B$, $j=A+1, \ldots, A+B$,

$$
\begin{gathered}
K_{i j}\left(h^{(i)}, h^{(j)}\right)(v, I)=\int_{\mathbb{R}^{3}} \int_{0}^{\infty} \int_{S^{2}} \int_{0}^{1} \int_{0}^{1} \frac{e^{-\frac{m_{j}}{2}\left|v_{*}\right|^{2}-I_{*}}}{\left(2 \pi / m_{j}\right)^{3 / 2}} \\
\times\left[h^{(j)}\left(v_{*}^{\prime},(1-r)(1-R) E\right)+h^{(i)}\left(v^{\prime}, r(1-R) E\right)\right. \\
\left.-h^{(j)}\left(v_{*}, I_{*}\right)-h^{(i)}(v, I)\right] \frac{T}{q_{j}(T)} B_{i j}\left(\sqrt{T} \sqrt{E}, \sqrt{T} R^{1 / 2}\left|v-v_{*}\right|, \frac{v-v_{*}}{\left|v-v_{*}\right|} \cdot \sigma\right) \\
\times(1-R) R^{1 / 2} \varphi_{i}(I)^{-1} d R d r d \sigma d I_{*} d v_{*},
\end{gathered}
$$

with $E=\frac{1}{2} \mu_{i j}\left|v-v_{*}\right|^{2}+I+I_{*}$ and $v^{\prime}, v_{*}^{\prime}$ are defined in (12), (13).

The Galilean invariance (isotropy in the space of velocities) can be seen on each of the operators $K_{i j}$. Namely for all isometric transformation $\mathcal{R}$ in $O(3, \mathbb{R})$, one has (denoting by $o$ the composition w.r.t the velocity variable only),

$$
K_{i j}\left(h^{(i)} \circ \mathcal{R}, h^{(j)} \circ \mathcal{R}\right)(v, I)=K_{i j}\left(h^{(i)}, h^{(j)}\right)(\mathcal{R} v, I) .
$$

We introduce now the scalar product that will be used throughout the paper. Given two vectors $k=\left(k^{(1)}, \ldots, k^{(A+B)}\right)$ and $\underline{l}=\left(l^{(1)}, \ldots, l^{(A+B)}\right)$, with $k^{(1)}, \ldots, k^{(A)}, l^{(1)}, \ldots, l^{(A)}$ functions of $V$, and $k^{(A+1)}, \ldots, k^{(A+B)}, l^{(A+1)}, \ldots, l^{(A+B)}$ functions of $V, J$, we define

$$
\langle\underline{k} \mid \underline{l}\rangle:=\sum_{i=1}^{A} n^{(i)} \int_{\mathbb{R}^{3}} \frac{e^{-m_{i} \frac{|V|^{2}}{2}}}{\left(2 \pi / m_{i}\right)^{3 / 2}} k^{(i)}(V) l^{(i)}(V) d V
$$




$$
+\sum_{i=A+1}^{A+B} n^{(i)} \int_{0}^{\infty} \int_{\mathbb{R}^{3}} \frac{\left.e^{-\left(m_{i} \frac{|V|^{2}}{2}\right.}+J\right)}{\left(2 \pi / m_{i}\right)^{3 / 2}} k^{(i)}(V, J) l^{(i)}(V, J) \frac{T \varphi_{i}(J T)}{q_{i}(T)} d V d J .
$$

We observe that the operator $\mathcal{K}$ defined in (19) is symmetric w.r.t. the scalar product $\langle\cdot \mid \cdot\rangle$, so that (admitting that it satisfies Fredholm's property, which we do here since we work at the formal level), its image is the orthogonal of its kernel.

We refer to [7], [12] and [9] for the Fredholm property in the case of a mixture of monoatomic gases (with the same or different masses).

The kernel $\mathbb{K}$ of $\mathcal{K}$ can easily be found (provided that all cross sections $B_{i j}$ are strictly positive). We refer for this to a computation done in [16].

It is constituted by the vectors $\underline{l}^{\Delta, j}(j=1, \ldots, A+B), \underline{l}^{U, z}(z=1,2,3)$ and $\underline{l}^{E}$, defined as

$$
\begin{gathered}
\underline{l}^{\Delta, j}=\left(\begin{array}{c}
l^{(1), \Delta, j} \\
\cdot \\
\cdot \\
l^{(j), \Delta, j} \\
\cdot \\
\cdot \\
\cdot \\
l^{(A+B), \Delta, j}
\end{array}\right)=\left(\begin{array}{c}
0 \\
0 \\
\cdot \\
0 \\
1 \\
0 \\
\cdot \\
0 \\
0
\end{array}\right), \\
\underline{l}^{U, z}=\left(\begin{array}{c}
l^{(1), U, z} \\
\cdot \\
l^{(A+B), U, z}
\end{array}\right)=\left(\begin{array}{c}
m_{1} V_{z} \\
\cdot \\
\cdot \\
m_{A+B} V_{z}
\end{array}\right), \\
\underline{l}^{E}=\left(\begin{array}{c}
m_{1} \frac{V^{2}}{2}+r_{1} J \\
\cdot \\
\cdot \\
l^{(1), E} \\
\cdot \\
l^{(A+B), E}
\end{array}\right)=\left(\begin{array}{c}
\frac{V^{2}}{2}+r_{A+B} J
\end{array}\right) .
\end{gathered}
$$

All of these properties will be useful for the description of the transport coefficients in the Navier-Stokes systems obtained in next section.

\section{Chapman-Enskog expansion FOR a miXture OF MONO- AND POLY- ATOMIC GASES}

We perform in this section the Chapman-Enskog expansion for a mixture of mono- and poly- atomic gases, when the collision operators are defined by the formulas developed in the previous section of this paper. The expansion is done at the formal level, we do not try here to present a functional setting which would be adapted for obtaining a rigorous expansion. We recall nevertheless that such a setting exists in the case of one single monoatomic gas (cf. [24]). 
3.1. Principle of the expansion. We present in this subsection the basic ideas underlying the Chapman-Enskog expansion. As in the previous section, we introduce a mixture of $A$ monoatomic gases and $B$ polyatomic gases. We systematically use the notations of subsections 2.1 to 2.3 .

We start by writing the Chapman-Enskog expansion for our mixture. We first define the rescaled (w.r.t the Knudsen number) system of Boltzmann equations:

$$
\partial_{t} f^{(i)}+v \cdot \nabla_{x} f^{(i)}=\frac{1}{\varepsilon} \sum_{j=1}^{A+B} Q_{i j}\left(f^{(i)}, f^{(j)}\right)
$$

where the operators $Q_{i j}$ are defined by formulas (1), (4), (11).

We then look for solutions of the Boltzmann equation (30) under the form

$$
f^{(i)}=M_{\varepsilon}^{(i)}\left(1+\varepsilon g_{\varepsilon}^{(i)}\right),
$$

where $M_{\varepsilon}^{(i)}$ is a Maxwellian distribution of (number) density $n_{\varepsilon}^{(i)}:=n_{\varepsilon}^{(i)}(t, x) \geq 0$, macroscopic velocity $u_{\varepsilon}:=u_{\varepsilon}(t, x) \in \mathbb{R}^{3}$, and temperature $T_{\varepsilon}:=T_{\varepsilon}(t, x) \geq 0$, that is (cf. (16))

$$
M_{\varepsilon}^{(i)}=\frac{n_{\varepsilon}^{(i)}}{\left(2 \pi T_{\varepsilon} / m_{i}\right)^{3 / 2} q_{i}\left(T_{\varepsilon}\right)} \exp \left(-\frac{m_{i}\left|v-u_{\varepsilon}\right|^{2}+2 r_{i} I}{2 T_{\varepsilon}}\right),
$$

with $r_{i}=0$ for $i=1, \ldots, A$ and $r_{i}=1$ for $i=A+1, \ldots, A+B$. We also assume (this is done without loss of generality, since one can perform a modification of the parameters of the Maxwellian distribution by adding terms of order $\varepsilon$, cf. [13] for example) that the vector of perturbed distributions $g=\left(g_{\varepsilon}^{(1)}, \ldots, g_{\varepsilon}^{(A+B)}\right)$, with functions $g_{\varepsilon}^{(i)}:=g_{\varepsilon}^{(i)}(t, x, v) \in \mathbb{R}$ for $i=1, \ldots, A$, and $g_{\varepsilon}^{(i)}:=g_{\varepsilon}^{(i)}(t, x, v, I) \in \mathbb{R}$ for $i=A+1, \ldots, A+B$, satisfies the conditions

$$
\begin{gathered}
\forall i=1, \ldots, A+B, \quad\left\langle\underline{g} \mid \underline{l}^{\Delta, i}\right\rangle=0, \\
\forall z=1, \ldots, 3, \quad\left\langle\underline{g} \mid \underline{l}^{U, z}\right\rangle=0, \\
\left\langle\underline{g} \mid \underline{l}^{E}\right\rangle=0,
\end{gathered}
$$

where $\langle\cdot \mid \cdot\rangle$ is the scalar product defined in (26) and vectors $\underline{l}^{\Delta, i}, \underline{l}^{U, z}, \underline{l}^{E}$ are provided in (27), (28), (29).

Introducing (31) in equation (30), we get the (approximated) system of linear equations satisfied by $g_{\varepsilon}^{(i)}$ for $i=1, \ldots, A+B$ :

$$
\begin{aligned}
\left(M_{\varepsilon}^{(i)}\right)^{-1}\left(\partial_{t} M_{\varepsilon}^{(i)}+v \cdot \nabla_{x} M_{\varepsilon}^{(i)}\right) & =\left(M_{\varepsilon}^{(i)}\right)^{-1} \sum_{j=1}^{A+B}\left[Q_{i j}\left(M_{\varepsilon}^{(i)}, M_{\varepsilon}^{(j)} g_{\varepsilon}^{(j)}\right)\right. \\
& \left.+Q_{i j}\left(M_{\varepsilon}^{(i)} g_{\varepsilon}^{(i)}, M_{\varepsilon}^{(j)}\right)\right] .
\end{aligned}
$$

Then for $i=1, \ldots, A$, thanks to (18),

$$
\left(M_{\varepsilon}^{(i)}\right)^{-1}\left(\partial_{t} M_{\varepsilon}^{(i)}+v \cdot \nabla_{x} M_{\varepsilon}^{(i)}\right)=\sum_{j=1}^{A} n_{\varepsilon}^{(j)} K_{i j}\left(v \mapsto g^{(i)}\left(v \sqrt{T_{\varepsilon}}+u_{\varepsilon}\right), v \mapsto g^{(j)}\left(v \sqrt{T_{\varepsilon}}+u_{\varepsilon}\right)\right)
$$




$$
+\sum_{j=A+1}^{A+B} n_{\varepsilon}^{(j)} K_{i j}\left(v \mapsto g^{(i)}\left(v \sqrt{T_{\varepsilon}}+u_{\varepsilon}\right),(v, I) \mapsto g^{(j)}\left(v \sqrt{T_{\varepsilon}}+u_{\varepsilon}, I T_{\varepsilon}\right)\right),
$$

and for $i=A+1, \ldots, A+B$, thanks to (18) again,

$$
\begin{gathered}
\left(M_{\varepsilon}^{(i)}\right)^{-1}\left(\partial_{t} M_{\varepsilon}^{(i)}+v \cdot \nabla_{x} M_{\varepsilon}^{(i)}\right) \\
=\sum_{j=1}^{A} n_{\varepsilon}^{(j)} K_{i j}\left((v, I) \mapsto g^{(i)}\left(v \sqrt{T_{\varepsilon}}+u_{\varepsilon}, I T_{\varepsilon}\right), v \mapsto g^{(j)}\left(v \sqrt{T_{\varepsilon}}+u_{\varepsilon}\right)\right) \\
+\sum_{j=A+1}^{A+B} n_{\varepsilon}^{(j)} K_{i j}\left((v, I) \mapsto g^{(i)}\left(v \sqrt{T_{\varepsilon}}+u_{\varepsilon}, I T_{\varepsilon}\right),(v, I) \mapsto g^{(j)}\left(v \sqrt{T_{\varepsilon}}+u_{\varepsilon}, I T_{\varepsilon}\right)\right),
\end{gathered}
$$

where the linear operators $K_{i j}$ are defined by $(21)-(23)$.

We can at this level write down the compressible Navier-Stokes equations (neglecting terms of order $\varepsilon^{2}$ ) of the mixture under the following abstract form:

- Mass conservation for each monoatomic species: $i=1, \ldots, A$,

$$
\partial_{t} \int_{\mathbb{R}^{3}} M_{\varepsilon}^{(i)} m_{i} d v+\nabla_{x} \cdot \int_{\mathbb{R}^{3}} M_{\varepsilon}^{(i)} m_{i} v d v=-\varepsilon \nabla_{x} \cdot \int_{\mathbb{R}^{3}} M_{\varepsilon}^{(i)} g_{\varepsilon}^{(i)} m_{i} v d v ;
$$

- Mass conservation for each polyatomic species: $i=A+1, \ldots, A+B$,

$$
\begin{gathered}
\partial_{t} \int_{\mathbb{R}^{3}} \int_{\mathbb{R}_{+}} M_{\varepsilon}^{(i)} m_{i} \varphi_{i}(I) d I d v+\nabla_{x} \cdot \int_{\mathbb{R}^{3}} \int_{\mathbb{R}_{+}} M_{\varepsilon}^{(i)} m_{i} v \varphi_{i}(I) d I d v \\
=-\varepsilon \nabla_{x} \cdot \int_{\mathbb{R}^{3}} \int_{\mathbb{R}_{+}} M_{\varepsilon}^{(i)} g_{\varepsilon}^{(i)} m_{i} v \varphi_{i}(I) d v d I
\end{gathered}
$$

- Momentum conservation of the mixture (we consider the components $k=$ $1, \ldots, 3)$ :

$$
\begin{aligned}
& \text { (41) } \partial_{t}\left(\sum_{i=1}^{A} \int_{\mathbb{R}^{3}} M_{\varepsilon}^{(i)} m_{i} v_{k} d v+\sum_{i=A+1}^{A+B} \int_{\mathbb{R}^{3}} \int_{\mathbb{R}_{+}} M_{\varepsilon}^{(i)} m_{i} v_{k} \varphi_{i}(I) d I d v\right) \\
& +\nabla_{x} \cdot\left(\sum_{i=1}^{A} \int_{\mathbb{R}^{3}} M_{\varepsilon}^{(i)} m_{i} v_{k} v d v+\sum_{i=A+1}^{A+B} \int_{\mathbb{R}^{3}} \int_{\mathbb{R}_{+}} M_{\varepsilon}^{(i)} m_{i} v_{k} v \varphi_{i}(I) d I d v\right) \\
& =-\varepsilon \nabla_{x} \cdot\left(\sum_{i=1}^{A} \int_{\mathbb{R}^{3}} M_{\varepsilon}^{(i)} g_{\varepsilon}^{(i)} m_{i} v_{k} v d v+\sum_{i=A+1}^{A+B} \int_{\mathbb{R}^{3}} \int_{\mathbb{R}_{+}} M_{\varepsilon}^{(i)} g_{\varepsilon}^{(i)} m_{i} v_{k} v \varphi_{i}(I) d I d v\right) ;
\end{aligned}
$$

- Total energy conservation of the mixture:

(42) $\partial_{t}\left(\sum_{i=1}^{A} \int_{\mathbb{R}^{3}} M_{\varepsilon}^{(i)} m_{i} \frac{|v|^{2}}{2} d v+\sum_{i=A+1}^{A+B} \int_{\mathbb{R}^{3}} \int_{\mathbb{R}_{+}} M_{\varepsilon}^{(i)}\left(m_{i} \frac{|v|^{2}}{2}+I\right) \varphi_{i}(I) d I d v\right)$

$$
\begin{aligned}
& +\nabla_{x} \cdot\left(\sum_{i=1}^{A} \int_{\mathbb{R}^{3}} M_{\varepsilon}^{(i)} m_{i} \frac{|v|^{2}}{2} v d v+\sum_{i=A+1}^{A+B} \int_{\mathbb{R}^{3}} \int_{\mathbb{R}_{+}} M_{\varepsilon}^{(i)}\left(m_{i} \frac{|v|^{2}}{2}+I\right) v \varphi_{i}(I) d I d v\right) \\
& =-\varepsilon \nabla_{x} \cdot\left(\sum_{i=1}^{A} \int_{\mathbb{R}^{3}} M_{\varepsilon}^{(i)} g_{\varepsilon}^{(i)} m_{i} \frac{|v|^{2}}{2} v d v+\sum_{i=A+1}^{A+B} \int_{\mathbb{R}^{3}} \int_{\mathbb{R}_{+}} M_{\varepsilon}^{(i)} g_{\varepsilon}^{(i)}\left(m_{i} \frac{|v|^{2}}{2}+I\right) v \varphi_{i}(I) d I d v\right) .
\end{aligned}
$$


Next subsections are devoted to computations enabling to write these abstract equations in such a way that they clearly appear as a system of compressible NavierStokes equations for our mixture (with dissipative terms of order $\varepsilon$, as always when Chapman-Enskog expansions are concerned). In subsection 3.2, we compute the l.h.s. of equations (39) - (42), which amounts to identifying the terms of order 0 in the expansion, corresponding to the system of compressible Euler equations for the mixture. Then subsection 3.3 is devoted to the computation of the r.h.s, of equations (39) - (42), which amounts to identifying the terms of order $\varepsilon$ in the expansion, corresponding to the dissipative terms in the system of compressible Navier-Stokes equations for our mixture.

3.2. Euler system. We present here as announced the computations for the l.h.s. of equations $(39)-(42)$. We denote

$$
\eta_{i}(T)=\int_{0}^{\infty} I \varphi_{i}(I) e^{-I / T} d I
$$

and do not write anymore the dependence w.r.t. $\varepsilon$ of the various considered terms. In the formalism of [21], [19], the term $\eta_{i}(T) / q_{i}(T)$ appearing in (50) corresponds to the average internal energy of the $i^{t h}$ species. We first compute moments relations for Maxwellian distributions (in the formulas below, components are labeled by $k, l=1, \ldots, 3)$ :

$\forall i=A+1, \ldots, A+B, \quad \int_{\mathbb{R}^{3}} \int_{\mathbb{R}_{+}} M^{(i)} m_{i} v_{k} v_{l} \varphi_{i}(I) d I d v=m_{i} n^{(i)} u_{k} u_{l}+n^{(i)} T \delta_{k l}$,

$$
\begin{gathered}
\forall i=1, \ldots, A, \quad \int_{\mathbb{R}^{3}} M^{(i)} m_{i} \frac{|v|^{2}}{2} d v=m_{i} n^{(i)} \frac{|u|^{2}}{2}+\frac{3}{2} n^{(i)} T, \\
\forall i=A+1, \ldots, A+B, \quad \int_{\mathbb{R}^{3}} \int_{\mathbb{R}_{+}} M^{(i)}\left(m_{i} \frac{|v|^{2}}{2}+I\right) \varphi_{i}(I) d I d v \\
=m_{i} n^{(i)} \frac{|u|^{2}}{2}+n^{(i)}\left[\frac{3}{2} T+\frac{\eta_{i}(T)}{q_{i}(T)}\right], \\
\forall i=1, \ldots, A, \quad \int_{\mathbb{R}^{3}} M^{(i)} m_{i} \frac{|v|^{2}}{2} v_{k} d v=m_{i} n^{(i)} \frac{|u|^{2}}{2} u_{k}+\frac{5}{2} n^{(i)} T u_{k},
\end{gathered}
$$




$$
\begin{aligned}
\forall i=A+1, \ldots, A+B, & \int_{\mathbb{R}^{3}} \int_{\mathbb{R}_{+}} M^{(i)}\left(m_{i} \frac{|v|^{2}}{2}+I\right) v_{k} \varphi_{i}(I) d I d v \\
& =m_{i} n^{(i)} \frac{|u|^{2}}{2} u_{k}+n^{(i)} u_{k}\left[\frac{5}{2} T+\frac{\eta_{i}(T)}{q_{i}(T)}\right] .
\end{aligned}
$$

Using identities (43) - (52), we get as announced the Euler system in conservative form (up to terms of order $\varepsilon$ ) (remember that we use for the components the notation $k=1, \ldots, 3)$ :

$$
i=1, \ldots, A+B, \quad \partial_{t}\left(m_{i} n^{(i)}\right)+\nabla_{x} \cdot\left(m_{i} n^{(i)} u\right)=0,
$$

$$
k=1, \ldots, 3, \quad \partial_{t}\left(\sum_{i=1}^{A+B} m_{i} n^{(i)} u_{k}\right)+\sum_{l} \partial_{x_{l}}\left(\sum_{i=1}^{A+B}\left[m_{i} n^{(i)} u_{k} u_{l}+n^{(i)} T \delta_{k l}\right]\right)=0,
$$

$$
\begin{gathered}
\partial_{t}\left(\sum_{i=1}^{A}\left[m_{i} n^{(i)} \frac{|u|^{2}}{2}+\frac{3}{2} n^{(i)} T\right]+\sum_{i=A+1}^{A+B}\left[m_{i} n^{(i)} \frac{|u|^{2}}{2}+n^{(i)}\left[\frac{3}{2} T+\frac{\eta_{i}(T)}{q_{i}(T)}\right]\right]\right) \\
+\sum_{l} \partial_{x_{l}}\left(\sum_{i=1}^{A}\left[m_{i} n^{(i)} \frac{|u|^{2}}{2} u_{l}+\frac{5}{2} n^{(i)} T u_{l}\right]\right. \\
\left.+\sum_{i=A+1}^{A+B}\left[m_{i} n^{(i)} \frac{|u|^{2}}{2} u_{l}+n^{(i)} u_{l}\left[\frac{5}{2} T+\frac{\eta_{i}(T)}{q_{i}(T)}\right]\right]\right)=0 .
\end{gathered}
$$

Note that in these equations, one could introduce the formation energy at zero temperature $e_{i}^{0}$ of the $i$-th species. This term would in fact be unavoidable in the equations if we also had considered chemically reactive collisions.

These equations can be rewritten under the following non conservative form, which is useful for the computation of the dissipative terms (of order $\varepsilon$ ) appearing in the Chapman-Enskog asymptotics:

$$
\begin{gathered}
i=1, \ldots, A+B, \quad \partial_{t} n^{(i)}+\left(u \cdot \nabla_{x}\right) n^{(i)}+n^{(i)} \nabla_{x} \cdot u=0, \\
k=1, \ldots, 3, \quad \partial_{t} u_{k}+\left(u \cdot \nabla_{x}\right) u_{k}+\frac{\sum_{i=1}^{A+B} \partial_{x_{k}}\left(n^{(i)} T\right)}{\sum_{i=1}^{A+B} m_{i} n^{(i)}}=0, \\
\partial_{t} T+\left(u \cdot \nabla_{x}\right) T+2 \Lambda(T) T \nabla_{x} \cdot u=0,
\end{gathered}
$$

with

$$
\Lambda(T)=\frac{\sum_{j=1}^{A+B} n^{(j)}}{3 \sum_{j=1}^{A+B} n^{(j)}+2 \sum_{j=A+1}^{A+B} n^{(j)}\left(\frac{\eta_{j}}{q_{j}}\right)^{\prime}(T)} .
$$

3.3. Navier-Stokes system. In this subsection, we provide the dissipative terms (viscosity, Soret and Dufour terms, etc.) of order $\varepsilon$ which are typical of the Chapman-Enskog asymptotics. 
3.3.1. Computation of the l.h.s of the linear equations (37), (38). We start with the quantity

$$
\left(M^{(i)}\right)^{-1}\left[\partial_{t} M^{(i)}+v \cdot \nabla_{x} M^{(i)}\right],
$$

which appears in the l.h.s. of (37), (38). Skipping all intermediate computations and using identities (56) - (58) to eliminate temporal derivatives, we finally get

$$
\begin{gathered}
=\frac{v-u}{\sqrt{T}} \cdot\left[\sqrt{T}\left(\frac{\nabla_{x} n^{(i)}}{n^{(i)}}-\frac{m_{i} \sum_{j=1}^{A+B} \nabla_{x} n^{(j)}}{\sum_{j=1}^{A+B} m_{j} n^{(j)}}\right)+\left(1-\frac{m_{i} \sum_{j=1}^{A+B} n^{(j)}}{\sum_{j=1}^{A+B} m_{j} n^{(j)}}\right) \frac{\nabla_{x} T}{\sqrt{T}}\right] \\
+P\left(\frac{v-u}{\sqrt{T}}\right): m_{i}\left(\frac{\nabla_{x} u+\nabla_{x} u^{T}}{2}\right) \\
+\left(\frac{m_{i}}{T}|v-u|^{2}\left(\frac{1}{3}-\Lambda(T)\right)-2 \frac{I}{T} r_{i} \Lambda(T)+2\left(\frac{3}{2}+r_{i} T \frac{q_{i}^{\prime}(T)}{q_{i}(T)}\right) \Lambda(T)-1\right)\left(\nabla_{x} \cdot u\right) \\
+\left(\frac{m_{i}}{2} \frac{|v-u|^{2}}{T}+r_{i} \frac{I}{T}-\left(\frac{5}{2}+r_{i} T \frac{q_{i}^{\prime}(T)}{q_{i}(T)}\right)\right) \frac{v-u}{\sqrt{T}} \cdot \frac{\nabla_{x} T}{\sqrt{T}},
\end{gathered}
$$

with

$$
P(v)=v \otimes v-\frac{1}{3}|v|^{2} I d
$$

We now wish to point out the specificities of the formulas above. First, the term in $P\left(\frac{v-u}{\sqrt{T}}\right)$ is identical to the same term in the case of one monoatomic gas. The term in $\frac{v-u}{\sqrt{T}}$ in the second term of identity (60) is typical of mixtures, it does not appear when only one gas is considered. The term involving $\nabla_{x} \cdot u$ appears only when at least one polyatomic gas is part of the mixture (since in a mixture of monoatomic gase, one has $\Lambda(T)=\frac{1}{3}$ ). Finally, the last term has a shape which depends on the monoatomic or polyatomic character of the species $i$. When $i \in\{1, \ldots, A\}$, we recover the usual term (sometimes denoted by $Q$ ) $\left(\frac{m_{i}}{2} \frac{|v-u|^{2}}{T}-\frac{5}{2}\right) \frac{v-u}{\sqrt{T}}$, which is typical of monoatomic gases.

3.3.2. Orthogonality properties. In order to solve the linear system (37), (38), taking into account (60), we need to use orthogonality properties.

We then introduce the following families (we indicate the dependences w.r.t. the components by indices $p=1, \ldots, 3$, and sometimes $q=1, \ldots, 3)$ :

$$
\begin{aligned}
& \underline{k}^{P, p, q}=\left(\begin{array}{c}
k^{(1), P, p, q} \\
\cdot \\
\dot{k^{(A+B), P, p, q}}
\end{array}\right)=\left(\begin{array}{c}
P_{p q}(V) m_{1} \\
\cdot \\
\cdot \\
P_{p q}(V) m_{A+B}
\end{array}\right), \\
& \underline{k}^{Q, p}=\left(\begin{array}{c}
k^{(1), Q, p} \\
\cdot \\
k^{(A+B), Q, p}
\end{array}\right)=\left(\begin{array}{c}
V_{p}\left(\frac{m_{1}}{2} V^{2}+r_{1} J-\left(\frac{5}{2}+r_{1} T \frac{q_{1}^{\prime}(T)}{q_{1}(T)}\right)\right) \\
\cdot \\
V_{p}\left(\frac{m_{A+B}}{2} V^{2}+r_{A+B} J-\left(\frac{5}{2}+r_{A+B} T \frac{q_{A+B}^{\prime}(T)}{q_{A+B}(T)}\right)\right)
\end{array}\right),
\end{aligned}
$$




$$
\underline{k}^{W}=\left(\begin{array}{c}
k^{(1), W} \\
\cdot \\
\dot{\cdot} \\
k^{(A+B), W}
\end{array}\right)=\left(\begin{array}{c}
s^{(1)} \cdot V \\
\cdot \\
\dot{\cdot} \\
s^{(A+B)} \cdot V
\end{array}\right),
$$

for all families of three-dimensional vectors $s^{(i)}=\left(s_{1}^{(i)}, \ldots, s_{3}^{(i)}\right)(i=1, \ldots, A+B)$ such that

$$
s^{(1)} n^{(1)}+\ldots+s^{(A+B)} n^{(A+B)}=0,
$$

and

$$
\underline{k}^{D}=\left(\begin{array}{c}
k^{(1), D} \\
\cdot \\
\dot{k^{(A+B), D}}
\end{array}\right)=\left(\begin{array}{c}
m_{1} V^{2}\left(\frac{1}{3}-\Lambda(T)\right)-2 r_{1} J \Lambda(T)+\left(3+2 r_{1} T \frac{q_{1}^{\prime}(T)}{q_{1}(T)}\right) \Lambda(T)-1 \\
\cdot \\
\cdot \\
m_{A+B} V^{2}\left(\frac{1}{3}-\Lambda(T)\right)-2 r_{A+B} J \Lambda(T)+\left(3+2 r_{A+B} T \frac{q_{A+B}^{\prime}(T)}{q_{A+B}(T)}\right) \Lambda(T)-1
\end{array}\right),
$$

where $\Lambda(T)$ has been defined in (59).

Moreover, by computing $q_{i}^{\prime}(T)$ defined in (17), we get $q_{i}^{\prime}(T)=\eta_{i}(T) / T^{2}$. Hence by setting $\bar{E}_{i}=\eta_{i}(T) / q_{i}(T)$, we get

$$
\begin{aligned}
k^{(i), Q, p} & =V_{p}\left(\frac{m_{i}}{2} V^{2}+r_{i} J-\left(\frac{5}{2}+r_{i} \frac{\bar{E}_{i}}{T}\right)\right), \\
k^{(i), D} & =2 r_{i} \Lambda(T)\left(\frac{\bar{E}_{i}}{T}-J\right)+2\left(\frac{1}{3}-\Lambda(T)\right)\left(m_{i} \frac{V^{2}}{2}-\frac{3}{2}\right) .
\end{aligned}
$$

Remembering that in the case of monoatomic mixtures, $\Lambda(T)=\frac{1}{3}$, we see that the term (63) is non-zero in the case of the presence of a polyatomic component. With these notations, the terms (62), (63) can be connected to terms appearing in [19], [21].

In the sequel, use will be made also of the global matrices, collecting all components for indices $p, q=1, \ldots, 3$, which will be denoted by $\underline{k}^{P}=\left(\underline{k}^{P, p, q}\right)_{p, q=1, \ldots, 3}$, $\underline{k}^{Q}=\left(\underline{k}^{Q, p}\right)_{p=1, \ldots, 3}$.

In these families, the first $A$ components only depend on $V$ (and not on $J$ ). Note also that the families $\underline{k}^{Q}$ and $\underline{k}^{D}$ depend on $T$.

One can check that the subspace

$$
\operatorname{Span}\left(\underline{k}^{P, p, q}, \underline{k}^{Q, p}, \underline{k}^{W}, \underline{k}^{D}\right)
$$

is orthogonal, with respect to the scalar product $\langle\cdot \mid \cdot\rangle$ defined in $(26)$, to the subspace

$$
\operatorname{Span}\left(\underline{l}^{\Delta, j}, \underline{l}^{U, z}, \underline{l}^{E}\right) .
$$

In the case of $\underline{k}^{P, p, q}$, it is a direct consequence of the evenness properties and of changes of variables of the type $\left(V_{1}, V_{2}, V_{3}\right) \rightarrow\left(V_{1}, V_{3}, V_{2}\right)$.

For $\underline{k}^{Q, p}$ and $\underline{k}^{W}$, the properties of evenness enable to consider only $\underline{l}^{U, z}$, and for $p=z$ only. This last case can be treated by a direct computation.

Finally for $\underline{k}^{D}$, one needs to perform a direct computation for $\underline{l}^{\Delta, j}$ and $\underline{l}^{E}$, the case of $\underline{l}^{U, z}$ being treated by evenness properties.

Still assuming the Fredholm property, notice that the families $\left(\underline{k}^{P, p, q}, \underline{k}^{Q, p}, \underline{k}^{W}, \underline{k}^{D}\right)$ belong to the image of $\mathcal{K}$ defined in (19), so that it is possible to find families of functions such that their image by $\mathcal{K}$ is one of the functions of $\left(\underline{k}^{P, p, q}, \underline{k}^{Q, p}, \underline{k}^{W}, \underline{k}^{D}\right)$. 
Moreover such a family of functions is unique if we also impose that it belongs to the orthogonal of the kernel of $\mathcal{K}$.

In other words, for all family of tridimensional vectors $s^{(i)}, i=1, \ldots, A+B$, such that the relation (61) is satisfied, we can find the functions

$$
\begin{gathered}
\underline{h}^{W}=\left(h^{(i), W}\right)_{i=1, \ldots, A+B}, \quad \underline{h}^{P, p, q}=\left(h^{(i), P, p, q}\right)_{i=1, \ldots, A+B}, \\
\underline{h}^{D}=\left(h^{(i), D}\right)_{i=1, \ldots, A+B}, \quad \underline{h}^{Q, p}=\left(h^{(i), Q, p}\right)_{i=1, \ldots, A+B}, \quad p, q=1 \ldots 3
\end{gathered}
$$

(whose components depend on $V$ for $i=1, \ldots, A$ and on $V, J$ for $i=A+1, \ldots, A+$ $B)$, satisfying the linear integral equations

(64) $\mathcal{K}\left(\underline{h}^{W}\right)=\underline{k}^{W}, \quad \mathcal{K}\left(\underline{h}^{P, p, q}\right)=\underline{k}^{P, p, q}, \quad \mathcal{K}\left(\underline{h}^{D}\right)=\underline{k}^{D}, \quad \mathcal{K}\left(\underline{h}^{Q, p}\right)=\underline{k}^{Q, p}$,

and the orthogonality relations:

$$
\begin{gathered}
\forall i=1, \ldots, A+B, \quad\left\langle\underline{h} \mid \underline{l}^{\Delta, i}\right\rangle=0, \\
\forall z=1, \ldots, 3, \quad\left\langle\underline{h} \mid \underline{l}^{U, z}\right\rangle=0, \\
\left\langle\underline{h} \mid \underline{l}^{E}\right\rangle=0,
\end{gathered}
$$

with a generic notation $\underline{h}=\underline{h}^{P, p, q}, \underline{h}^{Q, p}, \underline{h}^{W}, \underline{h}^{D}$.

3.3.3. Galilean invariance and computation of $g^{(i)}$. We now notice that thanks to the Galilean invariance (25), we can write (cf. [15]) for $i=1, \ldots, A$ :

$h^{(i), P, p, q}(V)=\tilde{h}^{(i), P}(|V|) P_{p q}(V), \quad h^{(i), Q, p}(V)=\tilde{h}^{(i), Q}(|V|) V_{p}, \quad h^{(i), D}(V)=\tilde{h}^{(i), D}(|V|)$,

and for $i=A+1, \ldots, A+B$ :

$$
\begin{gathered}
h^{(i), P, p, q}(V, J)=\tilde{h}^{(i), P}(|V|, J) P_{p q}(V), \quad h^{(i), Q, p}(V, J)=\tilde{h}^{(i), Q}(|V|, J) V_{p}, \\
h^{(i), D}(V, J)=\tilde{h}^{(i), D}(|V|, J) .
\end{gathered}
$$

Thanks to (37), (38), and the computations (60), we see that the previous definitions lead to the following formula for the perturbation $g^{(i)}$ :

$$
\begin{aligned}
i= & 1, \ldots, A \quad g^{(i)}(V \sqrt{T}+u)=\tilde{h}^{(i), P}(|V|) P(V):\left(\frac{\nabla_{x} u+\nabla_{x} u^{T}}{2}\right) \\
& +\tilde{h}^{(i), D}(|V|) \nabla_{x} \cdot u+\tilde{h}^{(i), Q}(|V|) V \cdot \frac{\nabla_{x} T}{\sqrt{T}}+\sqrt{T} h^{(i), W}(V),
\end{aligned}
$$

$$
\begin{gathered}
i=A+1, \ldots, A+B \quad g^{(i)}(V \sqrt{T}+u, J T)=\tilde{h}^{(i), P}(|V|, J) P(V):\left(\frac{\nabla_{x} u+\nabla_{x} u^{T}}{2}\right) \\
+\tilde{h}^{(i), D}(|V|, J) \nabla_{x} \cdot u+\tilde{h}^{(i), Q}(|V|, J) V \cdot \frac{\nabla_{x} T}{\sqrt{T}}+\sqrt{T} h^{(i), W}(V, J),
\end{gathered}
$$

with vectors $s^{(i)}$ appearing in $\underline{k}^{W}$ (thus affecting $h^{(i), W}$ ) provided by

$$
s^{(i)}=\frac{\nabla_{x} n^{(i)}}{n^{(i)}}-\frac{m_{i} \sum_{j=1}^{A+B} \nabla_{x} n^{(j)}}{\sum_{j=1}^{A+B} m_{j} n^{(j)}}+\left(1-\frac{m_{i} \sum_{j=1}^{A+B} n^{(j)}}{\sum_{j=1}^{A+B} m_{j} n^{(j)}}\right) \frac{\nabla_{x} T}{T} .
$$

Note that the vectors $s^{(i)}$ satisfy the relation (61). 
Note also that performing some elementary manipulations, $s^{(i)}$ can be rewritten as

$$
s^{(i)}=\frac{n}{n_{i}} d^{(i)}, \quad d^{(i)}=\nabla_{x}\left(\frac{p_{i}}{p}\right)+\left(\frac{p_{i}}{p}-\frac{\rho^{(i)}}{\sum_{j=1}^{A+B} \rho^{(j)}}\right) \frac{\nabla_{x} p}{p},
$$

with

$$
n=\sum_{i=1}^{A+B} n_{i}, \quad \rho_{i}=m_{i} n^{(i)}, \quad p_{i}=n^{(i)} T, \quad p=\sum_{i=1}^{A+B} p_{i},
$$

which is consistent with [19], [21]. The quantities $d^{(i)}$ are the diffusion driving forces.

3.3.4. Computation of the dissipative terms. We can then make explicit the computation of the diffusion terms in the Chapman-Enskog expansion, that is the quantities appearing as derivatives in the r.h.s of (39) - (42).

We begin by considering, for $i=1, \ldots, A$ and $k=1, \ldots, 3$ :

$$
D_{k}^{(i)}:=\int_{\mathbb{R}^{3}} M^{(i)} g^{(i)} m_{i} v_{k} d v .
$$

Hence by using a change of variables, we get

$$
\begin{aligned}
D_{k}^{(i)} & =\sqrt{T} n^{(i)} \int_{\mathbb{R}^{3}} \frac{e^{-m_{i} \frac{|V|^{2}}{2}}}{\left(2 \pi / m_{i}\right)^{3 / 2}} \tilde{h}^{(i), Q}(|V|) V \cdot \frac{\nabla_{x} T}{\sqrt{T}} m_{i} V_{k} d V \\
& +\sqrt{T} n^{(i)} \int_{\mathbb{R}^{3}} \frac{e^{-m_{i} \frac{|V|^{2}}{2}}}{\left(2 \pi / m_{i}\right)^{3 / 2}} h^{(i), W}(V) \sqrt{T} m_{i} V_{k} d V .
\end{aligned}
$$

Hence according to evenness properties, it comes that

$$
\begin{gathered}
D_{k}^{(i)}=n^{(i)} \int_{\mathbb{R}^{3}} \frac{e^{-\frac{|V|^{2}}{2}}}{(2 \pi)^{3 / 2}} \tilde{h}^{(i), Q}\left(\frac{|V|}{\sqrt{m_{i}}}\right) V_{1}^{2} d V \partial_{x_{k}} T \\
+T n^{(i)} m_{i} \int_{\mathbb{R}^{3}} \frac{e^{-m_{i} \frac{|V|^{2}}{2}}}{\left(2 \pi / m_{i}\right)^{3 / 2}} h^{(i), W}(V) V_{k} d V .
\end{gathered}
$$

In the same way, for $i=A+1, \ldots, A+B$ and $k=1, \ldots, 3$ :

$$
\begin{gathered}
D_{k}^{(i)}:=\int_{0}^{+\infty} \int_{\mathbb{R}^{3}} M^{(i)} g^{(i)} m_{i} v_{k} \varphi_{i}(I) d v d I \\
=n^{(i)} T \int_{0}^{+\infty} \int_{\mathbb{R}^{3}} \frac{e^{-\frac{|V|^{2}}{2}-J}}{(2 \pi)^{3 / 2}} \tilde{h}^{(i), Q}\left(\frac{|V|}{\sqrt{m_{i}}}, J\right) V_{1}^{2} \frac{\varphi_{i}(J T)}{q_{i}(T)} d V d J \partial_{x_{k}} T \\
+T^{2} n^{(i)} m_{i} \int_{0}^{+\infty} \int_{\mathbb{R}^{3}} \frac{e^{-m_{i} \frac{|V|^{2}}{2}-J}}{\left(2 \pi / m_{i}\right)^{3 / 2}} h^{(i), W}(V, J) V_{k} \frac{\varphi_{i}(J T)}{q_{i}(T)} d V d J .
\end{gathered}
$$

In the previous relation, the term $h^{(i), W}$ depends on a linear combination of the terms $s^{(i)}, i \in\{1 ; A+B\}$ defined in (67). Hence, we recover in this way the interspecies diffusion terms (Fick) and the terms corresponding to the Soret effect. 
We then compute, for $k, l=1, \ldots, 3$,

$$
F_{k l}:=\sum_{i=1}^{A} \int_{\mathbb{R}^{3}} M^{(i)} g^{(i)} m_{i} v_{k} v_{l} d v+\sum_{i=A+1}^{A+B} \int_{0}^{\infty} \int_{\mathbb{R}^{3}} M^{(i)} g^{(i)} m_{i} v_{k} v_{l} \varphi_{i}(I) d v d I .
$$

By using again a change of variable, and taking into account evenness properties and the fact that for any function $a:=a(|V|)$,

$$
\int_{\mathbb{R}^{3}} a(|V|)\left(V_{1}^{4}-V_{1}^{2} V_{2}^{2}\right) d V=2 \int_{\mathbb{R}^{3}} a(|V|) V_{1}^{2} V_{2}^{2} d V,
$$

we get

$$
\begin{gathered}
F_{k l}=\left(\sum_{i=1}^{A} T \frac{n^{(i)}}{m_{i}} \int_{\mathbb{R}^{3}} \frac{e^{-\frac{|V|^{2}}{2}}}{(2 \pi)^{3 / 2}} \tilde{h}^{(i), P}\left(\frac{|V|}{\sqrt{m_{i}}}\right) \frac{2}{3} V_{1}^{4} d V\right. \\
\left.+\sum_{i=A+1}^{A+B} T^{2} \frac{n^{(i)}}{m_{i}} \int_{0}^{\infty} \int_{\mathbb{R}^{3}} \frac{e^{-\frac{|V|^{2}}{2}-J}}{(2 \pi)^{3 / 2}} \tilde{h}^{(i), P}\left(\frac{|V|}{\sqrt{m_{i}}}, J\right) \frac{2}{3} V_{1}^{4} \frac{\varphi_{i}(J T)}{q_{i}(T)} d V d J\right) \\
\times\left[\frac{\nabla_{x} u+\nabla_{x} u^{T}}{2}-\frac{1}{3} \nabla_{x} \cdot u I d\right]_{k l} \\
+T \nabla_{x} \cdot u \delta_{k l}\left(\sum_{i=1}^{A} n^{(i)} \int_{\mathbb{R}^{3}} \frac{e^{-\frac{|V|^{2}}{2}}}{(2 \pi)^{3 / 2}} \tilde{h}^{(i), D}\left(\frac{|V|}{\sqrt{m_{i}}}\right) V_{1}^{2} d V\right. \\
\left.+\sum_{i=A+1}^{A+B} n^{(i)} T \int_{0}^{\infty} \int_{\mathbb{R}^{3}} \frac{e^{\frac{|V|^{2}}{2}}-J}{(2 \pi)^{3 / 2}} \tilde{h}^{(i), D}\left(\frac{|V|}{\sqrt{m_{i}}}, J\right) V_{1}^{2} \frac{\varphi_{i}(J T)}{q_{i}(T)} d V d J\right),
\end{gathered}
$$

so that viscosity terms are recovered.

We finally compute (for $k=1, \ldots, 3$ )

$$
G_{k}=\sum_{i=1}^{A} \int_{\mathbb{R}^{3}} M^{(i)} g^{(i)} m_{i} \frac{|v|^{2}}{2} v_{k} d v+\sum_{i=A+1}^{A+B} \int_{0}^{\infty} \int_{\mathbb{R}^{3}} M^{(i)} g^{(i)}\left(m_{i} \frac{|v|^{2}}{2}+I\right) v_{k} \varphi_{i}(I) d v d I .
$$

With the same change of variables as before and by using the expression of $g^{(i)}$ (cf. (65) and (66)) and evenness properties, we get

$$
\begin{gathered}
G_{k}=\sum_{l} F_{k l} u_{l}+T\left\{\sum_{i=1}^{A} \frac{n^{(i)}}{m_{i}} \int_{\mathbb{R}^{3}} \frac{e^{-\frac{|V|^{2}}{2}}}{(2 \pi)^{3 / 2}} \tilde{h}^{(i), Q}\left(\frac{|V|}{\sqrt{m_{i}}}\right) \frac{|V|^{2}}{2} V_{1}^{2} d V\right\} \partial_{x_{k}} T \\
+T^{2}\left\{\sum_{i=A+1}^{A+B} \frac{n^{(i)}}{m_{i}} \int_{0}^{\infty} \int_{\mathbb{R}^{3}} \frac{e^{-\frac{|V|^{2}}{2}}-J}{(2 \pi)^{3 / 2} q_{i}(T)} \tilde{h}^{(i), Q}\left(\frac{|V|}{\sqrt{m_{i}}}, J\right)\left(\frac{|V|^{2}}{2}+J\right) V_{1}^{2} \varphi_{i}(J T) d V d J\right\} \partial_{x_{k}} T \\
+T^{2}\left\{\sum_{i=1}^{A} n^{(i)} \int_{\mathbb{R}^{3}} \frac{e^{-m_{i} \frac{|V|^{2}}{2}}}{\left(2 \pi / m_{i}\right)^{3 / 2}} h^{(i), W}(V) m_{i} \frac{|V|^{2}}{2} V_{k} d V\right\} \\
+T^{3}\left\{\sum_{i=A+1}^{A+B} n^{(i)} \int_{0}^{\infty} \int_{\mathbb{R}^{3}} \frac{e^{-m_{i} \frac{|V|^{2}}{2}}-J}{\left(2 \pi / m_{i}\right)^{3 / 2} q_{i}(T)} h^{(i), W}(V, J)\left[m_{i} \frac{|V|^{2}}{2}+J\right]\right. \\
\left.\times V_{k} \varphi_{i}(J T) d V d J\right\} .
\end{gathered}
$$


In the previous formula, the terms $h^{(i), W}$ contain a linear combination of the gradients of the concentrations. Hence, this final computation shows the dissipative terms corresponding to the (Fourier) diffusion of temperature, and those related to the Dufour effect.

We finally write down the system (39) - (42) in the following semi-explicit form:

$$
i=1, \ldots, A+B \quad \partial_{t}\left(m_{i} n^{(i)}\right)+\nabla_{x} \cdot\left(m_{i} n^{(i)} u\right)=-\varepsilon \nabla_{x} \cdot D^{(i)}
$$

$$
\begin{gathered}
k=1, \ldots, 3 \quad \partial_{t}\left(\sum_{i=1}^{A+B} m_{i} n^{(i)} u_{k}\right)+\sum_{l} \partial_{x_{l}}\left(\sum_{i=1}^{A+B}\left[m_{i} n^{(i)} u_{k} u_{l}+n^{(i)} T \delta_{k l}\right]\right) \\
=-\varepsilon \sum_{l} \partial_{x_{l}} F_{k l},
\end{gathered}
$$

$$
\partial_{t}\left(\sum_{i=1}^{A}\left[m_{i} n^{(i)} \frac{|u|^{2}}{2}+\frac{3}{2} n^{(i)} T\right]+\sum_{i=A+1}^{A+B}\left[m_{i} n^{(i)} \frac{|u|^{2}}{2}+n^{(i)}\left[\frac{3}{2} T+\frac{\eta_{i}(T)}{q_{i}(T)}\right]\right]\right)
$$$$
+\sum_{l} \partial_{x_{l}}\left(\sum_{i=1}^{A}\left[m_{i} n^{(i)} \frac{|u|^{2}}{2} u_{l}+\frac{5}{2} n^{(i)} T u_{l}\right]+\sum_{i=A+1}^{A+B}\left[m_{i} n^{(i)} \frac{|u|^{2}}{2} u_{l}+n^{(i)} u_{l}\left[\frac{5}{2} T+\frac{\eta_{i}(T)}{q_{i}(T)}\right]\right]\right)
$$

$$
=-\varepsilon \nabla_{x} \cdot G
$$

In those equations, the terms $D^{(i)}, F_{k l}$ and $G$ are given by formulas $(69),(70)$, (71) and (72) in terms of the functions $\tilde{h}^{(i), Q}, h^{(i), W}, \tilde{h}^{(i), P}$ and $\tilde{h}^{(i), D}$.

Before writing comments about the above equations, we explain some symmetry properties between dissipative terms.

3.3.5. Symmetry of the Dufour et Soret terms. In this part, we perform a connection with the formalism developed in [19], [21]. By introducing the specific enthalpy of the $i^{t h}$ species $h_{i}$ by

$$
h_{i}=\left(\frac{5}{2} T+r_{i} \bar{E}_{i}\right) \frac{1}{m_{i}}
$$


$G_{k}$ writes

$$
\begin{aligned}
G_{k} & =\sum_{l=1}^{3} F_{k l} u_{l}+T \sum_{i=1}^{A} n^{(i)} \int_{\mathbb{R}^{3}} \frac{e^{-m_{i} \frac{V^{2}}{2}}}{\left(2 \pi / m_{i}\right)^{\frac{3}{2}}} h^{(i), Q, k}(V) k^{(i), Q, k}(V) d V \partial_{x_{k}} T \\
& +T \sum_{i=A+1}^{A+B} n^{(i)} \int_{\mathbb{R}^{3}} \int_{\mathbb{R}_{+}} \frac{e^{-m_{i} \frac{V^{2}}{2}-J}}{\left(2 \pi / m_{i}\right)^{\frac{3}{2}}} h^{(i), Q, k}(V, J) k^{(i), Q, k}(V, J) T \frac{\varphi_{i}(J T)}{q_{i}(T)} d V d J \partial_{x_{k}} T \\
& +T^{2} \sum_{i=1}^{A} n^{(i)} \int_{\mathbb{R}^{3}} \frac{e^{-m_{i} \frac{V^{2}}{2}}}{\left(2 \pi / m_{i}\right)^{\frac{3}{2}}} h^{(i), W}(V) k^{(i), Q, k}(V) d V \\
& +T^{2} \sum_{i=A+1}^{A+B} n^{(i)} \int_{\mathbb{R}^{3}} \int_{\mathbb{R}_{+}} \frac{e^{-m_{i} \frac{V^{2}}{2}-J}}{\left(2 \pi / m_{i}\right)^{\frac{3}{2}}} h^{(i), W}(V, J) k^{(i), Q, k}(V, J) T \frac{\varphi_{i}(J T)}{q_{i}(T)} d V d J \\
& +\sum_{i=1}^{A+B} h_{i} D_{k}^{(i)} .
\end{aligned}
$$

Using relations (64), we get

$$
G_{k}=\sum_{l=1}^{3} F_{k l} u_{l}+T\left\langle\mathcal{K}^{-1}\left(\underline{k}^{Q, k}\right), \underline{k}^{Q, k}\right\rangle \partial_{x_{k}} T+T^{2}\left\langle\mathcal{K}^{-1}\left(\underline{k}^{W}\right), \underline{k}^{Q, k}\right\rangle+\sum_{i=1}^{A+B} h_{i} D_{k}^{(i)} .
$$

Next, by using the symmetry of the linearized Boltzmann operator, one obtains

$$
\begin{aligned}
\left\langle\mathcal{K}^{-1}\left(\underline{k}^{W}\right), \underline{k}^{Q, k}\right\rangle & =\sum_{i=1}^{A} n^{(i)} \int_{\mathbb{R}^{3}} \frac{e^{-m_{i} \frac{V^{2}}{2}}}{\left(2 \pi / m_{i}\right)^{\frac{3}{2}}} s_{k}^{(i)} h^{(i), Q, k}(V) V_{k} d V \\
& +\sum_{i=A+1}^{A+B} n^{(i)} \int_{\mathbb{R}^{3}} \int_{\mathbb{R}_{+}} \frac{e^{-m_{i} \frac{V^{2}}{2}-J}}{\left(2 \pi / m_{i}\right)^{\frac{3}{2}}} s_{k}^{(i)} h^{(i), Q, k}(V, J) V_{k} T \frac{\varphi_{i}(J T)}{q_{i}(T)} d V d J .
\end{aligned}
$$

The first term of the right-hand side of this identity can be rewritten as

$$
n^{(i)} \int_{\mathbb{R}^{3}} \frac{e^{-m_{i} \frac{V^{2}}{2}}}{\left(2 \pi / m_{i}\right)^{\frac{3}{2}}} h^{(i), Q, k}(V) V_{k} d V=\left\langle\underline{h}^{Q, k}, b^{(i)}\right\rangle=\left\langle\underline{h}^{Q, k},\left(I d-P_{\mathbb{K}}\right) b^{(i)}\right\rangle,
$$

where $b^{(i)} \in \mathbb{R}^{A+B}$, and its components are defined by

$$
b_{j}^{(i)}:=V_{k} \delta_{i j}
$$

Moreover, $P_{\mathbb{K}}$ is the projection on the kernel of $\mathcal{K}$ defined by (27), (28) and (29).

In the same way, we write, for $i=A+1, \ldots, A+B$,

$n^{(i)} \int_{\mathbb{R}^{3}} \int_{\mathbb{R}_{+}} \frac{e^{-m_{i} \frac{V^{2}}{2}-J}}{\left(2 \pi / m_{i}\right)^{\frac{3}{2}}} h^{(i), Q, k}(V, J) V_{k} T \frac{\varphi_{i}(J T)}{q_{i}(T)} d V d J=\left\langle\underline{h}^{Q, k}, b^{(i)}\right\rangle=\left\langle\underline{h}^{Q, k}, \psi^{D_{i}}\right\rangle$,

with

$\psi^{D_{i}}:=\left(I d-P_{\mathbb{K}}\right) b^{(i)}, \quad \psi_{j}^{D_{i}}=\left(\delta_{i j}-\frac{n^{(i)} m_{j}}{\rho}\right) V_{k}, j=1 . . A+B, \quad \rho:=\sum_{j=1}^{A+B} \rho^{(j)}$.

Hence by setting

$$
\theta_{i}:=-\frac{T}{n^{(i)}}\left\langle\mathcal{K}^{-1}\left(\underline{k}^{Q, k}\right), \psi^{D_{i}}\right\rangle, \quad \lambda:=-T\left\langle\mathcal{K}^{-1}\left(\underline{k}^{Q, k}\right), \underline{k}^{Q, k}\right\rangle,
$$


we get

$$
G_{k}=\sum_{l=1}^{3} F_{k l} u_{l}-\lambda \partial_{x_{k}} T-p \sum_{i=1}^{A+B} \theta_{i} d^{(i)}+\sum_{i=1}^{A+B} h_{i} D_{k}^{(i)} .
$$

In this identity, the third term represents the Dufour effect.

Next we compute $D_{k}^{(i)}$ as

$$
D_{k}^{(i)}=m_{i} \partial_{x_{k}} T\left\langle\underline{h}^{Q, k}, \psi^{D_{i}}\right\rangle+m_{i} T\left\langle\underline{h}^{W}, \psi^{D_{i}}\right\rangle .
$$

Moreover, by using the definition $\underline{h}^{W}$ and the symmetry of $\mathcal{K}^{-1}$, we get

$$
\begin{aligned}
m_{i} T\left\langle\underline{h}^{W}, \psi^{D_{i}}\right\rangle & =m_{i} T \sum_{j=1}^{A+B} \frac{n}{n_{j}}\left\langle\psi^{D_{j}}, \mathcal{K}^{-1}\left(\psi^{D_{i}}\right)\right\rangle d^{(j)} \\
& =-\sum_{j=1}^{A+B} C_{j i} d^{(j)}
\end{aligned}
$$

with

$$
C_{j i}=-m_{i} T \frac{n}{n_{j}}\left\langle\psi^{D_{j}}, \mathcal{K}^{-1}\left(\psi^{D_{i}}\right)\right\rangle
$$

Therefore,

$$
D_{k}^{(i)}=-\rho_{i} \theta_{i} \partial_{x_{k}} \ln (T)-\sum_{j=1}^{A+B} C_{j i} d^{(j)} .
$$

The first term represents the Soret effect whereas the terms $C_{j i}$ correspond to the multicomponent flux diffusion coefficients. By comparison with the third term in (79), we recover the symmetry property between Soret et Dufour effects, cf. [19], [21].

3.4. Remarks on the result of the Chapman-Enskog computation. We explain here the main differences between the system of Navier-Stokes equations written in this work and the corresponding system for a mixture of monoatomic gases:

- First, the energy equation makes use of the internal energy $\frac{3}{2} T+\frac{\eta_{i}(T)}{q_{i}(T)}$ instead of $\frac{3}{2} T$ (this of course would already be seen at the level of Euler equations),

- Secondly, in the viscosity term $F_{k l}$, the second part (proportional to $\nabla_{x} \cdot u$ ) only appears when polyatomic gases are considered (cf. comments at the end of subsection 3.3.1).

\section{Explicit computations in the CASE OF CONSTANT CROSS SECTions}

The quantities $h^{(i)}$ which appear in the definition of $g^{(i)}$ and therefore in the dissipative quantities $D_{k}^{(i)}, F_{k l}$ and $G_{k}$ (which are part of the Navier-Stokes system of compressible monoatomic and polyatomic gas mixtures) cannot in general be explicitly computed.

As in the case of a single monotaomic gas, it is however possible to compute them when the cross sections (here denoted by $B_{i j}$ ) appearing in the collision operators $Q_{i j}$ are very simple. Therefore, in this section, we shall systematically use the assumption that $B_{i j}$ is constant (and $B_{i j}=B_{j i}$ ). Moreover, in order to be 
coherent with the fact that in the air, the main polyatomic species (that is, $\mathrm{O}_{2}$ and $N_{2}$ ) are in fact diatomic, we also shall assume that for all $i=A+1, \ldots, A+B$, one has $\varphi_{i}(I)=1$, and $q_{i}(T)=T$.

In that specific case, the quantities $\underline{k}^{W}, \underline{k}^{P}, \underline{k}^{D}$, and $\underline{k}^{Q}$ can be written in the following way:

$$
\begin{gathered}
k^{(i), W}=s^{(i)} V, \quad k^{(i), P, p, q}=m_{i} P_{p q}(V), \\
k^{(i), Q, p}=\left(m_{i} \frac{|V|^{2}}{2}-\frac{5}{2}\right) V_{p}+r_{i}(J-1) V_{p}, \\
k^{(i), D}=\left(m_{i}|V|^{2}-3\right)\left(\frac{1}{3}-\Lambda\right)-2 r_{i} \Lambda(J-1) .
\end{gathered}
$$

Note that using the formalism of [33], [27] and [18], they can be rewritten as

$$
\begin{gathered}
k^{(i), W}=s^{(i)} \phi_{i}^{1000 i}, \quad k^{(i), P, p, q}=\left.\phi_{i}^{2000 i}\right|_{p q}, \\
k^{(i), Q, p}=\left.\phi_{i}^{1010 i}\right|_{p}+\left.r_{i} \phi_{i}^{1001 i}\right|_{p}, \quad k^{(i), D}=2\left(\frac{1}{3}-\Lambda\right) \phi_{i}^{0010 i}-2 r_{i} \Lambda \phi_{i}^{0001 i} .
\end{gathered}
$$

In the case when the $B_{i j}$ are not constant (and even if they only depend upon $\left.\frac{v-v_{*}}{\left|v-v_{*}\right|} \cdot \sigma\right)$, one needs to solve the linear (spatially homogeneous) integral problems $\mathcal{K}\left(\underline{h}^{W}\right)=\underline{k}^{W}$, etc., together with suitable orthogonality conditions. This can of course be done only at the numerical level. It requires then a lot of care in the effective implementation and a significant amount of computational time, even after the isotropy properties of the kernels have been exploited.

The next four subsections are respectively devoted to the computation of $\underline{h}^{W}$, $\underline{h}^{P}=\left(\underline{h}^{P, p, q}\right)_{p, q=1, \ldots, 3}, \underline{h}^{D}$ and $\underline{h}^{Q}=\left(\underline{h}^{Q, p}\right)_{p=1, \ldots, 3}$. Then, subsection 4.5 contains the computation of $D_{k}^{(i)}, F_{k l}$ and $G_{k}$, starting from the values obtained for $\underline{h}^{W}, \underline{h}^{P}$, $\underline{h}^{D}$ and $\underline{h}^{Q}$. In the procedure, use will be made of integrals reported in Appendix A. Since these computations are quite long, only the main results are displayed in subsections 4.1 to 4.4. Detailed computations can be found in Appendix B.

4.1. Computation of $\underline{h}^{W}$. We want to solve the problem

$$
\mathcal{K}\left(\underline{h}^{W}\right)=\underline{k}^{W},
$$

with

$$
k^{(i), W}=s^{(i)} \cdot V,
$$

and with the orthogonality constraints

$$
\begin{gathered}
\forall i=1, \ldots, A+B, \quad\left\langle\underline{h}^{W} \mid \underline{l}^{\Delta, i}\right\rangle=0, \\
\forall z=1, \ldots, 3, \quad\left\langle\underline{h}^{W} \mid \underline{l}^{U, z}\right\rangle=0, \\
\left\langle\underline{h}^{W} \mid \underline{l}^{E}\right\rangle=0
\end{gathered}
$$

(with scalar product defined in (26) and $\underline{l}^{\Delta, i}, \underline{\underline{b}}^{U, z}, \underline{l}^{E}$ defined in (27), (28), (29)).

We test for that the effect of $K_{i j}$ on combinations of $m_{i} v_{1}$. Skipping all details (that the interested reader may find in Appendix B), we finally get

$$
K_{i j}\left(v \mapsto W_{1}^{(i)} m_{i} v_{1}, v \mapsto W_{1}^{(j)} m_{j} v_{1}\right)=\tilde{B}_{i j} \mu_{i j}\left(W_{1}^{(j)}-W_{1}^{(i)}\right) v_{1},
$$


where $W_{1}^{(i)}, W_{1}^{(j)} \in \mathbb{R}$ are constants, and $\tilde{B}_{i j}$ are defined in terms of the constant cross sections $B_{i j}$ as

$$
\tilde{B}_{i j}=\left\{\begin{array}{lcc}
B_{i j} & \text { if } & i, j=1, \ldots, A, \\
\frac{2}{3} B_{i j} & \text { if } & i=1, \ldots, A, j=A+1, \ldots, A+B, \\
\frac{2}{3} B_{i j} & \text { if } & i=A+1, \ldots, A+B, j=1, \ldots, A, \\
\frac{4}{15} B_{i j} & \text { if } & i=A+1, \ldots, A+B, j=A+1, \ldots, A+B .
\end{array}\right.
$$

Therefore, after exchanging the variable 1 with any of the variables $p$, we get the solution

$$
\begin{gathered}
h^{(i), W}(V)=m_{i} W^{(i)} \cdot V, \quad i=1, \ldots, A, \\
h^{(i), W}(V, J)=m_{i} W^{(i)} \cdot V, \quad i=A+1, \ldots, A+B,
\end{gathered}
$$

where the tridimensional constants $W^{(i)}$ must satisfy the system

$$
\begin{gathered}
\sum_{j=1}^{A+B} n^{(j)} \tilde{B}_{i j} \mu_{i j}\left(W^{(j)}-W^{(i)}\right)=s^{(i)} \\
\sum_{i=1}^{A+B} m_{i} n^{(i)} W^{(i)}=0 .
\end{gathered}
$$

Note that the first part of the system only contains $A+B-1$ independent equations. It can be solved only under the constraint $\sum_{i=1}^{A+B} n^{(i)} s^{(i)}=0$.

We finish the computation by noticing that in the special case of a mixture of two gases, the system above can be solved very easily (remember that $\tilde{B}_{12}=\tilde{B}_{21}$ ):

with

$$
\begin{aligned}
W^{(1)} & =\frac{m_{2} s^{(1)}}{\left(m_{1} n^{(1)}+m_{2} n^{(2)}\right) \tilde{B}_{12} \mu_{12}}, \\
W^{(2)} & =\frac{-m_{1} s^{(2)}}{\left(m_{1} n^{(1)}+m_{2} n^{(2)}\right) \tilde{B}_{12} \mu_{12}},
\end{aligned}
$$

$$
\begin{aligned}
& s^{(1)}=\frac{\nabla_{x} n^{(1)}}{n^{(1)}}-\frac{m_{1}\left(\nabla_{x} n^{(1)}+\nabla_{x} n^{(2)}\right)}{m_{1} n^{(1)}+m_{2} n^{(2)}}+\left(1-\frac{m_{1}\left(n^{(1)}+n^{(2)}\right)}{m_{1} n^{(1)}+m_{2} n^{(2)}}\right) \frac{\nabla_{x} T}{T}, \\
& s^{(2)}=\frac{\nabla_{x} n^{(2)}}{n^{(2)}}-\frac{m_{2}\left(\nabla_{x} n^{(1)}+\nabla_{x} n^{(2)}\right)}{m_{1} n^{(1)}+m_{2} n^{(2)}}+\left(1-\frac{m_{2}\left(n^{(1)}+n^{(2)}\right)}{m_{1} n^{(1)}+m_{2} n^{(2)}}\right) \frac{\nabla_{x} T}{T} .
\end{aligned}
$$

4.2. Computation of $\underline{h}^{P}$. We now solve the problem

$$
\mathcal{K}\left(\underline{h}^{P, p, q}\right)=\underline{k}^{P, p, q}
$$

with (for each $p, q=1, \ldots, 3$ )

$$
\begin{gathered}
\forall i=1, \ldots, A+B, \quad\left\langle\underline{h}^{P, p, q} \mid \underline{l}^{\Delta, i}\right\rangle=0, \\
\forall z=1, \ldots, 3, \quad\left\langle\underline{h}^{P, p, q} \mid \underline{\underline{l}}^{U, z}\right\rangle=0, \\
\left\langle\underline{h}^{P, p, q} \mid \underline{l}^{E}\right\rangle=0 .
\end{gathered}
$$

We recall that

$$
k^{(i), P, p, q}=P_{p q}(V) m_{i} .
$$

The computation of $\underline{h}^{P}$ follows the same lines as the computation of $\underline{h}^{W}$. We consider the component $p=1, q=2$ of the tensor. With the notations of the 
previous paragraph for $\tilde{B}_{i j}$, for $i=1, \ldots, A+B, j=1, \ldots, A+B$, and $\Pi_{12}^{(i)}, \Pi_{12}^{(j)}$ real constants, we get (all details are reported in Appendix B):

$$
K_{i j}\left(v \mapsto m_{i} \Pi_{12}^{(i)} v_{1} v_{2}, v \mapsto m_{j} \Pi_{12}^{(j)} v_{1} v_{2}\right)=\tilde{B}_{i j} \mu_{i j}^{2}\left(\frac{\Pi_{12}^{(j)}-2 \Pi_{12}^{(i)}}{m_{j}}-\frac{\Pi_{12}^{(i)}}{m_{i}}\right) v_{1} v_{2} .
$$

All other components of the tensor can be treated in the same way, thanks to the isotropy properties of $\mathcal{K}$.

The solution to the problem (85) is thus

$$
\begin{gathered}
h^{(i), P, p, q}(V)=m_{i} \Pi_{12}^{(i)} P_{p, q}^{(i)}(V), \quad i=1, \ldots, A, \\
h^{(i), P, p, q}(V, J)=m_{i} \Pi_{12}^{(i)} P_{p, q}^{(i)}(V), \quad i=A+1, \ldots, A+B,
\end{gathered}
$$

where the constants $\Pi_{12}^{(i)}$ are defined by the system

$$
\sum_{j=1}^{A+B} n^{(j)} \tilde{B}_{i j} \mu_{i j}^{2}\left(\frac{\Pi_{12}^{(j)}-2 \Pi_{12}^{(i)}}{m_{j}}-\frac{\Pi_{12}^{(i)}}{m_{i}}\right)=m_{i} .
$$

We denote from now on $\Pi^{(i)}:=\Pi_{12}^{(i)}$. Using the Galilean invariance again, we get $h^{(i), P}(V)=\tilde{h}^{(i), P}(|V|) P(|V|)$, with

$$
\begin{gathered}
\tilde{h}^{(i), P}(|V|)=m_{i} \Pi^{(i)}, \quad i=1, \ldots, A, \\
\tilde{h}^{(i), P}(|V|, J)=m_{i} \Pi^{(i)}, \quad i=A+1, \ldots, A+B .
\end{gathered}
$$

4.3. Computation of $\underline{h}^{D}$. We recall that we consider here a mixture of monoatomic and diatomic gases, so that $\varphi_{i}(I)=1$ for $i=A+1, \ldots, A+B$. Then, the quantity $\Lambda(T)$ defined in (59) simplifies very much, and turns out to be independent of $T$ :

$$
\Lambda=\frac{\sum_{j=1}^{A+B} n^{(j)}}{3 \sum_{j=1}^{A+B} n^{(j)}+2 \sum_{j=A+1}^{A+B} n^{(j)}} .
$$

Note that for a completely monoatomic mixture, $\Lambda=\frac{1}{3}$, while for a completely diatomic mixture, $\Lambda=\frac{1}{5}$.

We have to solve the problem

$$
\mathcal{K}\left(\underline{h}^{D}\right)=\underline{k}^{D}
$$

where

$$
k^{(i), D}=\left(m_{i}|V|^{2}-3\right)\left(\frac{1}{3}-\Lambda\right)-2 r_{i} \Lambda(J-1),
$$

with

$$
\begin{gathered}
\forall i=1, \ldots, A+B, \quad\left\langle\underline{h}^{D} \mid \underline{l}^{\Delta, i}\right\rangle=0, \\
\forall z=1, \ldots, 3, \quad\left\langle\underline{h}^{D} \mid \underline{\underline{l}}^{U, z}\right\rangle=0, \\
\left\langle\underline{h}^{D} \mid \underline{l}^{E}\right\rangle=0 .
\end{gathered}
$$

In each computation of this subsection, the objective will be to try to cast the final results as proper combinations of $m_{i}|v|^{2}-3$ and $I-1$. We will skip intermediate steps, which are detailed in Appendix B.

We introduce indeterminate constant coefficients $\Delta^{(i)}$ for $i=1, \ldots, A+B$ and $\widetilde{\Delta}^{(i)}$ for $i=A+1, \ldots, A+B$. 
- $\quad$ For $i=1, \ldots, A$ and $j=1, \ldots, A$, we get

$$
\begin{aligned}
B_{i j}^{-1} K_{i j}(v & \left.\mapsto \Delta^{(i)}\left(m_{i}|v|^{2}-3\right), \quad v \mapsto \Delta^{(j)}\left(m_{j}|v|^{2}-3\right)\right)(v)= \\
& =-\frac{2 \mu_{i j}}{m_{i}+m_{j}}\left(\Delta^{(i)}-\Delta^{(j)}\right)\left(m_{i}|v|^{2}-3\right) .
\end{aligned}
$$

- $\quad$ For $i=1, \ldots, A$ and $j=A+1, \ldots, A+B$, we get

$$
\begin{gathered}
B_{i j}^{-1} K_{i j}\left(v \mapsto \Delta^{(i)}\left(m_{i}|v|^{2}-3\right), \quad(v, I) \mapsto \Delta^{(j)}\left(m_{j}|v|^{2}-3\right)+\widetilde{\Delta}^{(j)}(I-1)\right)(v)= \\
=-\frac{2}{15} \frac{m_{j}}{m_{i}+m_{j}}\left[2 \Delta^{(i)} \frac{5 m_{i}+m_{j}}{m_{i}+m_{j}}-\Delta^{(j)} \frac{8 m_{i}}{m_{i}+m_{j}}-\widetilde{\Delta}^{(j)}\right]\left(m_{i}|v|^{2}-3\right) .
\end{gathered}
$$

- For $i=A+1, \ldots, A+B$ and $j=1, \ldots, A$, we get

$$
\begin{aligned}
& B_{i j}^{-1} K_{i j}((v, I)\left.\mapsto \Delta^{(i)}\left(m_{i}|v|^{2}-3\right)+\widetilde{\Delta}^{(i)}(I-1), \quad v \mapsto \Delta^{(j)}\left(m_{j}|v|^{2}-3\right)\right)(v, I)= \\
&=-\frac{2}{15} \frac{m_{j}}{m_{i}+m_{j}}\left[2 \Delta^{(i)} \frac{5 m_{i}+m_{j}}{m_{i}+m_{j}}-\Delta^{(j)} \frac{8 m_{i}}{m_{i}+m_{j}}-\widetilde{\Delta}^{(i)}\right]\left(m_{i}|v|^{2}-3\right) \\
&+\frac{2}{5}\left(\Delta^{(i)} \frac{2 m_{j}}{m_{i}+m_{j}}+\Delta^{(j)} \frac{2 m_{i}}{m_{i}+m_{j}}-\widetilde{\Delta}^{(i)}\right)(I-1) .
\end{aligned}
$$

- For $i=A+1, \ldots, A+B$ and $j=A+1, \ldots, A+B$, we get

$$
\begin{aligned}
& B_{i j}^{-1} K_{i j}\left((v, I) \mapsto \Delta^{(i)}\left(m_{i}|v|^{2}-3\right)+\widetilde{\Delta}^{(i)}(I-1), \quad(v, I) \mapsto \Delta^{(j)}\left(m_{j}|v|^{2}-3\right)+\widetilde{\Delta}^{(j)}(I-1)\right)(v, I)= \\
& =-\frac{4}{105} \frac{m_{j}}{m_{i}+m_{j}}\left[\Delta^{(i)} \frac{2\left(7 m_{i}+2 m_{j}\right)}{m_{i}+m_{j}}-\Delta^{(j)} \frac{10 m_{i}}{m_{i}+m_{j}}-\widetilde{\Delta}^{(i)}-\widetilde{\Delta}^{(j)}\right]\left(m_{i}|v|^{2}-3\right) \\
& +\frac{8}{105}\left(\Delta^{(i)} \frac{3 m_{j}}{m_{i}+m_{j}}+\Delta^{(j)} \frac{3 m_{i}}{m_{i}+m_{j}}-\frac{5}{2} \widetilde{\Delta}^{(i)}+\widetilde{\Delta}^{(j)}\right)(I-1) .
\end{aligned}
$$

In conclusion, system (87) can be rewritten as :

- for $i=1, \ldots, A$,

$$
\begin{aligned}
\sum_{j=1}^{A} 2 n^{(j)} B_{i j} \frac{\mu_{i j}}{m_{i}+m_{j}}\left(\Delta^{(j)}-\Delta^{(i)}\right) & \\
+\sum_{j=A+1}^{A+B} n^{(j)} B_{i j} & \left\{\frac{16}{15} \frac{m_{j}}{m_{i}+m_{j}} \frac{m_{i}}{m_{i}+m_{j}} \Delta^{(j)}+\frac{2}{15} \frac{m_{j}}{m_{i}+m_{j}} \widetilde{\Delta}^{(j)}\right. \\
- & \left.\frac{4}{3} \frac{m_{j}\left(m_{i}+\frac{1}{5} m_{j}\right)}{\left(m_{i}+m_{j}\right)^{2}} \Delta^{(i)}\right\}=\frac{1}{3}-\Lambda,
\end{aligned}
$$


- for $i=A+1, \ldots, A+B$,

(89)

$$
\begin{gathered}
\sum_{j=1}^{A} n^{(j)} B_{i j}\left\{\frac{16}{15} \frac{\mu_{i j}}{m_{i}+m_{j}} \Delta^{(j)}-\frac{4}{15} \frac{m_{j}}{m_{i}+m_{j}} \frac{5 m_{i}+m_{j}}{m_{i}+m_{j}} \Delta^{(i)}+\frac{2}{15} \frac{m_{j}}{m_{i}+m_{j}} \widetilde{\Delta}^{(i)}\right\} \\
+\sum_{j=A+1}^{A+B} n^{(j)} B_{i j}\left\{\frac{8}{21} \frac{m_{i} m_{j}}{\left(m_{i}+m_{j}\right)^{2}} \Delta^{(j)}+\frac{4}{105} \frac{m_{j}}{m_{i}+m_{j}} \widetilde{\Delta}^{(j)}-\frac{4}{15} \frac{m_{j}\left(2 m_{i}+\frac{4}{7} m_{j}\right)}{\left(m_{i}+m_{j}\right)^{2}} \Delta^{(i)}\right. \\
\left.+\frac{4}{105} \frac{m_{j}}{m_{i}+m_{j}} \widetilde{\Delta}^{(i)}\right\}=\frac{1}{3}-\Lambda,
\end{gathered}
$$

$$
\begin{gathered}
\sum_{j=1}^{A} n^{(j)} B_{i j}\left\{\frac{4}{5} \frac{m_{i}}{m_{i}+m_{j}} \Delta^{(j)}+\frac{4}{5} \frac{m_{j}}{m_{i}+m_{j}} \Delta^{(i)}-\frac{2}{5} \widetilde{\Delta}^{(i)}\right\} \\
+\sum_{j=A+1}^{A+B} n^{(j)} B_{i j}\left\{\frac{8}{35} \frac{m_{i}}{m_{i}+m_{j}} \Delta^{(j)}+\frac{8}{105} \widetilde{\Delta}^{(j)}+\frac{8}{35} \frac{m_{j}}{m_{i}+m_{j}} \Delta^{(i)}-\frac{4}{21} \widetilde{\Delta}^{(i)}\right\}=-2 \Lambda .
\end{gathered}
$$

We can note that a suitable combination of the right hand sides of the system (88)-(90) vanishes. One can check that the same linear combination vanishes when one considers the left hand sides of the equations (88)-(90), yielding that one of these $A+2 B$ equations is redundant (more precisely, exactly $A+2 B-1$ equations are independent).

The solution to problem (87) is therefore

$$
\begin{aligned}
& h^{(i), D}(V)=\Delta^{(i)}\left(m_{i}|V|^{2}-3\right), \quad i=1, \ldots, A, \\
& h^{(i), D}(V, J)=\Delta^{(i)}\left(m_{i}|V|^{2}-3\right)+\widetilde{\Delta}^{(i)}(J-1), \quad i=A+1, \ldots, A+B,
\end{aligned}
$$

which, as expected from the Galilean invariance, depends on the vector $V$ only through its modulus $|V|$. The coefficients $\Delta^{(i)}, \widetilde{\Delta}^{(i)}$ are defined by any $A+2 B-1$ among the equations (88)-(90).

4.4. Computation of $\underline{h}^{Q}$. We recall that we wish to solve the problem

$$
\mathcal{K}\left(\underline{h}^{Q, p}\right)=\underline{k}^{Q, p},
$$

where

$$
k^{(i), Q, p}=\frac{1}{2}\left(m_{i}|V|^{2}-5\right) V_{p}+r_{i}(J-1) V_{p},
$$

with (for each $p=1, \ldots, 3$ )

$$
\begin{gathered}
\forall i=1, \ldots, A+B, \quad\left\langle\underline{h}^{Q, p} \mid \underline{l}^{\Delta, i}\right\rangle=0, \\
\forall z=1, \ldots, 3, \quad\left\langle\underline{h}^{Q, p} \mid \underline{l}^{U, z}\right\rangle=0, \\
\left\langle\underline{h}^{Q, p} \mid \underline{l}^{E}\right\rangle=0 .
\end{gathered}
$$

We test for that the effect of $K_{i j}$ on combinations of $|v|^{2} v_{1}$ and $v_{1}$. We introduce indeterminate constant coefficients $Q^{(i)}$ for $i=1, \ldots, A+B$ and $\tilde{Q}^{(i)}$ for $i=$ $A+1, \ldots, A+B$.

- For $i=1, \ldots, A$ and $j=1, \ldots, A$, we get

$$
B_{i j}^{-1} K_{i j}\left(v \mapsto Q^{(i)} m_{i}|v|^{2} v_{1}, \quad v \mapsto Q^{(j)} m_{j}|v|^{2} v_{1}\right)(v)=
$$




$$
\begin{gathered}
=Q^{(j)} \frac{m_{i}}{\left(m_{i}+m_{j}\right)^{3}}\left[\frac{8}{3} m_{i}^{2} m_{j}|v|^{2} v_{1}+5\left(m_{i}^{2}+m_{j}^{2}-\frac{2}{3} m_{i} m_{j}\right) v_{1}\right] \\
+Q^{(i)} \frac{m_{i}}{\left(m_{i}+m_{j}\right)^{3}}\left[-m_{j}\left(3 m_{i}^{2}+m_{j}^{2}+\frac{4}{3} m_{i} m_{j}\right)|v|^{2} v_{1}+10 m_{j}\left(m_{i}-\frac{1}{3} m_{j}\right) v_{1}\right] .
\end{gathered}
$$

From the computations relevant to $\underline{h}^{W}$ (see Subsection 4.1), we easily get:

$$
B_{i j}^{-1} K_{i j}\left(v \mapsto Q^{(i)} v_{1}, \quad v \mapsto Q^{(j)} v_{1}\right)(v)=\frac{1}{m_{i}+m_{j}}\left(m_{i} Q^{(j)}-m_{j} Q^{(i)}\right) v_{1} .
$$

Thus, combining the two previous results, we finally get

$$
\begin{gathered}
B_{i j}^{-1} K_{i j}\left(v \mapsto Q^{(i)}\left(m_{i}|v|^{2}-5\right) v_{1}, \quad v \mapsto Q^{(j)}\left(m_{j}|v|^{2}-5\right) v_{1}\right)(v)= \\
=\left\{Q^{(j)} \frac{8}{3} m_{i}^{2}-Q^{(i)}\left(3 m_{i}^{2}+\frac{4}{3} m_{i} m_{j}+m_{j}^{2}\right)\right\} \frac{m_{j}}{\left(m_{i}+m_{j}\right)^{3}}\left(m_{i}|v|^{2}-5\right) v_{1} .
\end{gathered}
$$

- For $i=1, \ldots, A$ and $j=A+1, \ldots, A+B$, we get

$$
\begin{aligned}
& B_{i j}^{-1} K_{i j}\left(v \mapsto Q^{(i)} m_{i}|v|^{2} v_{1}, \quad(v, I) \mapsto Q^{(j)} m_{j}|v|^{2} v_{1}+\widetilde{Q}^{(j)} I v_{1}\right)(v)= \\
& =Q^{(j)} \frac{2}{3} \frac{m_{i}}{\left(m_{i}+m_{j}\right)^{3}}\left[2 m_{i}^{2} m_{j}|v|^{2} v_{1}+5\left(m_{i}^{2}+m_{j}^{2}\right) v_{1}\right]+\widetilde{Q}^{(j)} \frac{2}{3} \frac{m_{i}^{2}}{\left(m_{i}+m_{j}\right)^{2}}\left[\frac{1}{5} m_{j}|v|^{2} v_{1}+v_{1}\right] \\
& +Q^{(i)} \frac{2}{3} \frac{m_{i} m_{j}}{\left(m_{i}+m_{j}\right)^{3}}\left[-\left(3 m_{i}^{2}+2 m_{i} m_{j}+m_{j}^{2}\right)|v|^{2} v_{1}+10 m_{i} v_{1}\right] .
\end{aligned}
$$

From Subsection 4.1, we easily get

$$
B_{i j}^{-1} K_{i j}\left(v \mapsto Q^{(i)} v_{1}, \quad v \mapsto Q^{(j)} v_{1}\right)(v)=\frac{2}{3} \frac{1}{m_{i}+m_{j}}\left(m_{i} Q^{(j)}-m_{j} Q^{(i)}\right) v_{1}
$$

(and this allows to compute also $\left.K_{i j}\left(v \mapsto 0, \quad v \mapsto-\widetilde{Q}^{(j)} v_{1}\right)(v)\right)$. Thus, combining the results, we obtain

$$
\begin{gathered}
B_{i j}^{-1} K_{i j}\left(v \mapsto Q^{(i)}\left(m_{i}|v|^{2}-5\right) v_{1}, \quad v \mapsto Q^{(j)}\left(m_{j}|v|^{2}-5\right) v_{1}+\widetilde{Q}^{(j)}(I-1) v_{1}\right)(v)= \\
=\left\{2 Q^{(j)} m_{i}-Q^{(i)}\left(3 m_{i}+2 m_{j}+\frac{m_{j}^{2}}{m_{i}}\right)+\frac{1}{5} \widetilde{Q}^{(j)}\left(m_{i}+m_{j}\right)\right\} \frac{2}{3} \frac{m_{i} m_{j}}{\left(m_{i}+m_{j}\right)^{3}}\left(m_{i}|v|^{2}-5\right) v_{1} .
\end{gathered}
$$

- $\quad$ For $i=A+1, \ldots, A+B$ and $j=1, \ldots, A$, we get

$$
\begin{aligned}
& B_{i j}^{-1} K_{i j}\left((v, I) \mapsto Q^{(i)} m_{i}|v|^{2} v_{1}+\widetilde{Q}^{(i)} I v_{1}, \quad v \mapsto Q^{(j)} m_{j}|v|^{2} v_{1}\right)(v, I)= \\
= & Q^{(j)} \frac{m_{i}}{\left(m_{i}+m_{j}\right)^{3}}\left[\frac{4}{3} m_{i}^{2} m_{j}|v|^{2} v_{1}+\frac{4}{3} m_{i}\left(m_{i}+m_{j}\right) I v_{1}+2\left(m_{i}^{2}-\frac{2}{3} m_{i} m_{j}+\frac{5}{3} m_{j}^{2}\right) v_{1}\right] \\
+ & Q^{(i)} \frac{m_{i} m_{j}}{\left(m_{i}+m_{j}\right)^{3}}\left[-\left(2 m_{i}^{2}+\frac{4}{3} m_{i} m_{j}+\frac{2}{3} m_{j}^{2}\right)|v|^{2} v_{1}+\frac{4}{3}\left(m_{i}+m_{j}\right) I v_{1}+\frac{4}{3}\left(4 m_{i}-m_{j}\right) v_{1}\right] \\
+ & \widetilde{Q}^{(i)} \frac{2}{15} \frac{1}{\left(m_{i}+m_{j}\right)^{2}}\left[m_{i}^{2} m_{j}|v|^{2} v_{1}-\left(3 m_{i}^{2}+8 m_{i} m_{j}+5 m_{j}^{2}\right) I v_{1}+\left(3 m_{i}-2 m_{j}\right) m_{i} v_{1}\right] .
\end{aligned}
$$


From Subsection 4.1, we have

$$
B_{i j}^{-1} K_{i j}\left(v \mapsto Q^{(i)} v_{1}, \quad v \mapsto Q^{(j)} v_{1}\right)(v)=\frac{2}{3} \frac{1}{m_{i}+m_{j}}\left(m_{i} Q^{(j)}-m_{j} Q^{(i)}\right) v_{1},
$$

therefore

$$
\begin{gathered}
B_{i j}^{-1} K_{i j}\left(v \mapsto Q^{(i)}\left(m_{i}|v|^{2}-5\right) v_{1}+\widetilde{Q}^{(i)}(I-1) v_{1}, \quad v \mapsto Q^{(j)}\left(m_{j}|v|^{2}-5\right) v_{1}\right)(v, I)= \\
=\left\{\frac{2}{3} Q^{(j)} m_{i}^{2}-Q^{(i)}\left(m_{i}^{2}+\frac{2}{3} m_{i} m_{j}+\frac{1}{3} m_{j}^{2}\right)+\frac{1}{15} \widetilde{Q}^{(i)} m_{i}\left(m_{i}+m_{j}\right)\right\} \\
\times 2 \frac{m_{j}}{\left(m_{i}+m_{j}\right)^{3}}\left(m_{i}|v|^{2}-5\right) v_{1} \\
+\left\{2 Q^{(j)} m_{i}^{2}+2 Q^{(i)} m_{i} m_{j}-\frac{1}{5} \widetilde{Q}^{(i)}\left(3 m_{i}^{2}+8 m_{i} m_{j}+5 m_{j}^{2}\right)\right\} \frac{2}{3} \frac{1}{\left(m_{i}+m_{j}\right)^{2}}(I-1) v_{1} .
\end{gathered}
$$

- $\quad$ For $i=A+1, \ldots, A+B$ and $j=A+1, \ldots, A+B$, we get

$$
\begin{aligned}
& B_{i j}^{-1} K_{i j}\left((v, I) \mapsto Q^{(i)} m_{i}|v|^{2} v_{1}+\widetilde{Q}^{(i)} I v_{1}, \quad(v, I) \mapsto Q^{(j)} m_{j}|v|^{2} v_{1}+\widetilde{Q}^{(j)} I v_{1}\right)(v, I)= \\
& =Q^{(j)} \frac{m_{i}}{\left(m_{i}+m_{j}\right)^{3}}\left[\frac{16}{35} m_{i}^{2} m_{j}|v|^{2} v_{1}+\frac{8}{21} m_{i}\left(m_{i}+m_{j}\right) I v_{1}+\left(\frac{20}{21} m_{i}^{2}+\frac{4}{3} m_{j}^{2}\right) v_{1}\right] \\
& \quad+\widetilde{Q}^{(j)} \frac{8}{105} \frac{m_{i}}{\left(m_{i}+m_{j}\right)^{2}}\left[\frac{1}{2} m_{i} m_{j}|v|^{2} v_{1}+\left(m_{i}+m_{j}\right) I v_{1}+\frac{5}{2} m_{i} v_{1}\right] \\
& +Q^{(i)} \frac{m_{i}}{\left(m_{i}+m_{j}\right)^{3}}\left[-\frac{4}{5} m_{j}\left(m_{i}^{2}+\frac{16}{21} m_{i} m_{j}+\frac{1}{3} m_{j}^{2}\right)|v|^{2} v_{1}+\frac{8}{21} m_{j}\left(m_{i}+m_{j}\right) I v_{1}+\frac{16}{7} m_{i} m_{j} v_{1}\right] \\
& +\widetilde{Q}^{(i)} \frac{8}{105} \frac{1}{\left(m_{i}+m_{j}\right)^{2}}\left[\frac{1}{2} m_{i}^{2} m_{j}|v|^{2} v_{1}-\frac{1}{2}\left(5 m_{i}+7 m_{j}\right)\left(m_{i}+m_{j}\right) I v_{1}+\frac{5}{2} m_{i}^{2} v_{1}\right] .
\end{aligned}
$$

From Subsection 4.1, we have

$$
B_{i j}^{-1} K_{i j}\left(v \mapsto Q^{(i)} v_{1}, \quad v \mapsto Q^{(j)} v_{1}\right)(v)=\frac{4}{15} \frac{1}{m_{i}+m_{j}}\left(m_{i} Q^{(j)}-m_{j} Q^{(i)}\right) v_{1},
$$

therefore

$$
\begin{aligned}
& B_{i j}^{-1} K_{i j}\left(v \mapsto Q^{(i)}\left(m_{i}|v|^{2}-5\right) v_{1}+\widetilde{Q}^{(i)}(I-1) v_{1}, \quad v \mapsto Q^{(j)}\left(m_{j}|v|^{2}-5\right) v_{1}+\widetilde{Q}^{(j)}(I-1) v_{1}\right)(v, I)= \\
& =\left\{\frac{4}{7} Q^{(j)} m_{i}^{2}-Q^{(i)}\left(m_{i}^{2}+\frac{16}{21} m_{i} m_{j}+\frac{1}{3} m_{j}^{2}\right)+\frac{1}{21}\left(\widetilde{Q}^{(j)}+\widetilde{Q}^{(i)}\right) m_{i}\left(m_{i}+m_{j}\right)\right\} \\
& \times \frac{4}{5} \frac{m_{j}}{\left(m_{i}+m_{j}\right)^{3}}\left(m_{i}|v|^{2}-5\right) v_{1} \\
& +\left\{Q^{(j)} m_{i}^{2}+Q^{(i)} m_{i} m_{j}+\frac{1}{5}\left(m_{i}+m_{j}\right)\left(\widetilde{Q}^{(j)} m_{i}-\frac{1}{2} \widetilde{Q}^{(i)}\left(5 m_{i}+7 m_{j}\right)\right)\right\} \frac{8}{21} \frac{1}{\left(m_{i}+m_{j}\right)^{2}}(I-1) v_{1} .
\end{aligned}
$$

In conclusion, the solution of problem (81) is thus

$$
\begin{aligned}
& h^{(i), Q, p}(V)=Q^{(i)}\left(m_{i}|V|^{2}-5\right) V_{p}, \quad i=1, \ldots, A, \\
& h^{(i), Q, p}(V, J)=Q^{(i)}\left(m_{i}|V|^{2}-5\right) V_{p}+\widetilde{Q}^{(i)}(J-1) V_{p}, \quad i=A+1, \ldots, A+B,
\end{aligned}
$$


and we note that, as already anticipated owing to the Galilean invariance, $h^{(i), Q, p}(V, J)=$ $\tilde{h}^{(i), Q}(|V|, J) V_{p}$, where

$$
\begin{aligned}
& \tilde{h}^{(i), Q}(|V|)=Q^{(i)}\left(m_{i}|V|^{2}-5\right), \quad i=1, \ldots, A, \\
& \tilde{h}^{(i), Q}(|V|, J)=Q^{(i)}\left(m_{i}|V|^{2}-5\right)+\widetilde{Q}^{(i)}(J-1), \quad i=A+1, \ldots, A+B .
\end{aligned}
$$

The coefficients $Q^{(i)}$ and $\widetilde{Q}^{(i)}$ are defined as solutions of the system

- for $i=1, \ldots, A$,

$$
\begin{aligned}
\sum_{j=1}^{A} n^{(j)} B_{i j}\left\{Q^{(j)} \frac{8}{3} m_{i}^{2}-Q^{(i)}\left(3 m_{i}^{2}+\frac{4}{3} m_{i} m_{j}+m_{j}^{2}\right)\right\} \frac{m_{j}}{\left(m_{i}+m_{j}\right)^{3}} \\
+\sum_{j=A+1}^{A+B} n^{(j)} B_{i j}\left\{2 Q^{(j)} m_{i}-Q^{(i)}\left(3 m_{i}+2 m_{j}+\frac{m_{j}^{2}}{m_{i}}\right)+\frac{1}{5} \widetilde{Q}^{(j)}\left(m_{i}+m_{j}\right)\right\} \\
\times \frac{2}{3} \frac{m_{i} m_{j}}{\left(m_{i}+m_{j}\right)^{3}}=\frac{1}{2},
\end{aligned}
$$

- for $i=A+1, \ldots, A+B$,

$\sum_{j=1}^{A} n^{(j)} B_{i j}\left\{\frac{2}{3} Q^{(j)} m_{i}^{2}-Q^{(i)}\left(m_{i}^{2}+\frac{2}{3} m_{i} m_{j}+\frac{1}{3} m_{j}^{2}\right)+\frac{1}{15} \widetilde{Q}^{(i)} m_{i}\left(m_{i}+m_{j}\right)\right\} 2 \frac{m_{j}}{\left(m_{i}+m_{j}\right)^{3}}$

$+\sum_{j=A+1}^{A+B} n^{(j)} B_{i j}\left\{\frac{4}{7} Q^{(j)} m_{i}^{2}-Q^{(i)}\left(m_{i}^{2}+\frac{16}{21} m_{i} m_{j}+\frac{1}{3} m_{j}^{2}\right)+\frac{1}{21}\left(\widetilde{Q}^{(j)}+\widetilde{Q}^{(i)}\right) m_{i}\left(m_{i}+m_{j}\right)\right\}$

$$
\times \frac{4}{5} \frac{m_{j}}{\left(m_{i}+m_{j}\right)^{3}}=\frac{1}{2}
$$

$\sum_{j=1}^{A} n^{(j)} B_{i j}\left\{2 Q^{(j)} m_{i}^{2}+2 Q^{(i)} m_{i} m_{j}-\frac{1}{5} \widetilde{Q}^{(i)}\left(3 m_{i}^{2}+8 m_{i} m_{j}+5 m_{j}^{2}\right)\right\} \frac{2}{3} \frac{1}{\left(m_{i}+m_{j}\right)^{2}}$

$+\sum_{j=A+1}^{A+B} n^{(j)} B_{i j}\left\{Q^{(j)} m_{i}^{2}+Q^{(i)} m_{i} m_{j}+\frac{1}{5}\left(m_{i}+m_{j}\right)\left(\widetilde{Q}^{(j)} m_{i}-\frac{1}{2} \widetilde{Q}^{(i)}\left(5 m_{i}+7 m_{j}\right)\right)\right\}$

$$
\times \frac{8}{21} \frac{1}{\left(m_{i}+m_{j}\right)^{2}}=1 .
$$

4.5. Obtention of the viscosity coefficients in the case of constant cross sections. We recall here the Navier-Stokes system obtained at the end of subsection 3.3.4: now write down eqs. (73) - (75) in the special case which is considered here:

$$
i=1, \ldots, A+B, \quad \partial_{t}\left(m_{i} n^{(i)}\right)+\nabla_{x} \cdot\left(m_{i} n^{(i)} u\right)=-\varepsilon \nabla_{x} \cdot D^{(i)},
$$

$$
k=1, \ldots, 3, \quad \partial_{t}\left(\sum_{i=1}^{A+B} m_{i} n^{(i)} u_{k}\right)+\sum_{l} \partial_{x_{l}}\left(\sum_{i=1}^{A+B}\left[m_{i} n^{(i)} u_{k} u_{l}+n^{(i)} T \delta_{k l}\right]\right)
$$




$$
=-\varepsilon \sum_{l} \partial_{x_{l}} F_{k l}
$$

$$
\begin{gathered}
+\sum_{l} \partial_{x_{l}}\left(\sum_{i=1}^{A}\left[m_{i} n^{(i)} \frac{|u|^{2}}{2} u_{l}+\frac{5}{2} n^{(i)} T u_{l}\right]+\sum_{i=A+1}^{A+B}\left[m_{i} n^{(i)} \frac{|u|^{2}}{2} u_{l}+\frac{7}{2} n^{(i)} T u_{l}\right]\right) \\
=-\varepsilon \nabla_{x} \cdot G .
\end{gathered}
$$

The viscous terms $D_{k}^{(i)}$ (for $i=1, \ldots, A+B$ ), $F_{k l}$ and $G_{k}$, have been computed in Subsection 3.3.4, in formulas (69) - (72).

When the cross sections $B_{i j}$ are constant, the functions $h^{(i), W}, \tilde{h}^{(i), P}, \tilde{h}^{(i), D}$ and $\tilde{h}^{(i), Q}$ have been computed in subsections 4.1 to 4.4 and may be cast in compact form, for $i=1, \ldots, A+B$, as

$$
\begin{aligned}
& h^{(i), W}=m_{i} W^{(i)} \cdot V, \\
& \tilde{h}^{(i), P}=m_{i} \Pi^{(i)}, \\
& \tilde{h}^{(i), D}=\Delta^{(i)}\left(m_{i}|V|^{2}-3\right)+r_{i} \widetilde{\Delta}^{(i)}(J-1), \\
& \tilde{h}^{(i), Q}=Q^{(i)}\left(m_{i}|V|^{2}-5\right)+r_{i} \widetilde{Q}^{(i)}(J-1),
\end{aligned}
$$

where the constant coefficients $W^{(i)}, \Pi^{(i)}, \Delta^{(i)}, \widetilde{\Delta}^{(i)}, Q^{(i)}, \widetilde{Q}^{(i)}$ fulfil suitable linear systems pointed out in subsections 4.1 to 4.4 .

The integrals appearing in coefficients $D_{k}^{(i)}, F_{k l}$ and $G_{k}$ can be then computed owing, whenever necessary, to integrals reported in Appendix A, and bearing in mind that we are assuming here that $\varphi_{i}(I)=1$, so that $q_{i}(T)=T$. Skipping all intermediate computations, viscosity terms turn out to be

$$
D_{k}^{(i)}=m_{i} W_{k}^{(i)} n^{(i)} T, \quad i=1, \ldots, A+B,
$$

where coefficients $W_{k}^{(i)}$ are combinations of the quantities $s^{(i)}$, (so that they contain gradients of number densities and of temperature), while

$F_{k l}=2 T \sum_{i=1}^{A+B} n^{(i)} \Pi^{(i)}\left[\frac{\nabla_{x} u+\nabla_{x} u^{T}}{2}-\frac{1}{3} \nabla_{x} \cdot u I d\right]_{k l}+2 T \nabla_{x} \cdot u \delta_{k l} \sum_{i=1}^{A+B} n^{(i)} \Delta^{(i)}$, and finally

$$
G_{k}=\sum_{l=1}^{3} F_{k l} u_{l}+T \partial_{x_{k}} T \sum_{i=1}^{A+B} \frac{n^{(i)}}{m_{i}}\left(5 Q^{(i)}+r_{i} \widetilde{Q}^{(i)}\right)+T^{2} \sum_{i=1}^{A+B} n^{(i)}\left(\frac{5}{2}+r_{i}\right) W_{k}^{(i)},
$$

where $\Pi^{(i)}, \Delta^{(i)}, W^{(i)}, Q^{(i)}$, and $\widetilde{Q}^{(i)}$ are computed in subsections 4.1 to 4.4. Note that $F_{k l}$ may be cast as

$$
F_{k l}=-\mu\left[\frac{\nabla_{x} u+\nabla_{x} u^{T}}{2}-\frac{1}{3} \nabla_{x} \cdot u I d\right]_{k l}-\kappa \nabla_{x} \cdot u \delta_{k l}
$$

where

$$
\mu=-2 T \sum_{i=1}^{A+B} n^{(i)} \Pi^{(i)}
$$


represents the shear viscosity, while the term $-\kappa \nabla_{x} \cdot u$ is the so-called dynamical pressure, an additional contribution to the classical scalar pressure which is typical of the polyatomic structure of molecules $[31,5]$. The bulk viscosity $\kappa$ is provided by the formula

$$
\kappa=-2 T \sum_{i=1}^{A+B} n^{(i)} \Delta^{(i)}
$$

Moreover, $G_{k}$ can be written as

$$
G_{k}=\sum_{l=1}^{3} F_{k l} u_{l}-\lambda \partial_{x_{k}} T+T^{2} \sum_{i=1}^{A+B} n^{(i)}\left(\frac{5}{2}+r_{i}\right) W_{k}^{(i)},
$$

where

$$
\lambda=-T \sum_{i=1}^{A+B} \frac{n^{(i)}}{m_{i}}\left(5 Q^{(i)}+r_{i} \widetilde{Q}^{(i)}\right)
$$

is the partial thermal conductivity, while the last term provides the dependence of the heat flux w.r.t. the gradients of number densities (through coefficients $W^{(i)}$ ).

It is also possible to rewrite $G_{k}$ within the formalism of equation (79). Indeed, in the Maxwellian case, $\bar{E}_{i}=T$, so that $h_{i}=\left(\frac{5}{2}+r_{i}\right) \frac{T}{m_{i}}$. Therefore, by using the expression $(96)$ of $D_{k}^{(i)}$, we see that

$$
G_{k}=\sum_{l=1}^{3} F_{k l} u_{l}-\lambda \partial_{x_{k}} T+\sum_{i=1}^{A+B} h_{i} D_{k}^{(i)} .
$$

Hence by comparison with (79), we see that the Dufour effect is equal to zero in the case of constant cross sections. Moreover, by using the relations (79), (80), we also see that the Soret effect is equal to 0 in this case.

To conclude, the system of Navier-Stokes equations which are obtained by the Chapman-Enskog procedure from a system of Boltzmann equations corresponding to a mixture of monoatomic and polyatomic gases with constant cross sections, can be written down explicitly thanks to equations $(92)-(94)$, together with formulas (96) - (98), and the linear finite-dimensional systems defined in subsections 4.1 to 4.4 .

Acknowledgement: The research leading to this paper was partially funded by Université Sorbonne Paris Cité, in the framework of the "Investissements d'Avenir", convention ANR-11-IDEX-0005; it also was partially funded by University of Parma and INdAM-GNFM.

\section{Appendix A: Some integrals}

Integrals over the variable $R$ :

$$
\begin{gathered}
\int_{0}^{1} R^{1 / 2} d R=\frac{2}{3}, \quad \int_{0}^{1}(1-R) R^{1 / 2} d R=\frac{4}{15} \\
\int_{0}^{1}(1-R) R^{3 / 2} d R=\frac{4}{35}, \quad \int_{0}^{1}(1-R)^{2} R^{1 / 2} d R=\frac{16}{105} .
\end{gathered}
$$


Integrals over the angular variable:

$$
\int_{S^{2}} d \sigma=1, \quad \int_{S^{2}}|\sigma|^{2} d \sigma=1, \quad \int_{S^{2}}\left(\sigma_{1}\right)^{2} d \sigma=\frac{1}{3} .
$$

Integrals over the velocity variable:

$$
\begin{array}{cc}
\int_{\mathbb{R}^{3}} \frac{e^{-\frac{1}{2}\left|v_{*}\right|^{2}}}{(2 \pi)^{3 / 2}} d v_{*}=1, \quad \int_{\mathbb{R}^{3}}\left|v_{*}\right|^{2} \frac{e^{-\frac{1}{2}\left|v_{*}\right|^{2}}}{(2 \pi)^{3 / 2}} d v_{*}=3, \\
\int_{\mathbb{R}^{3}}\left|v_{*}\right|^{4} \frac{e^{-\frac{1}{2}\left|v_{*}\right|^{2}}}{(2 \pi)^{3 / 2}} d v_{*}=15, \quad \int_{\mathbb{R}^{3}}\left|v_{*}\right|^{6} \frac{e^{-\frac{1}{2}\left|v_{*}\right|^{2}}}{(2 \pi)^{3 / 2}} d v_{*}=105,
\end{array}
$$

or, in case of particle masses $m_{i} \neq 1$,

$$
\left(\frac{m_{i}}{2 \pi}\right)^{3 / 2} \int_{\mathbb{R}^{3}} e^{-\frac{1}{2} m_{i}\left|v_{*}\right|^{2}} d v_{*}=1, \quad\left(\frac{m_{i}}{2 \pi}\right)^{3 / 2} \int_{\mathbb{R}^{3}} m_{i}\left|v_{*}\right|^{2} e^{-\frac{1}{2} m_{i}\left|v_{*}\right|^{2}} d v_{*}=3 .
$$

Use will be made also of some of the following relations:

$$
\begin{gathered}
\int_{\mathbb{R}^{3}}\left(v_{1}\right)^{2} A(|v|) d v=\frac{1}{3} \int_{\mathbb{R}^{3}}|v|^{2} A(|v|) d v, \\
\int_{\mathbb{R}^{3}}\left(v_{1}\right)^{4} A(|v|) d v=\frac{1}{5} \int_{\mathbb{R}^{3}}|v|^{4} A(|v|) d v, \\
\int_{\mathbb{R}^{3}}\left(v_{1}\right)^{2}\left(v_{2}\right)^{2} A(|v|) d v=\frac{1}{15} \int_{\mathbb{R}^{3}}|v|^{4} A(|v|) d v, \\
\int_{\mathbb{R}^{3}}\left(v_{1}\right)^{6} A(|v|) d v=\frac{1}{7} \int_{\mathbb{R}^{3}}|v|^{6} A(|v|) d v, \\
\int_{\mathbb{R}^{3}}\left(v_{1}\right)^{4}\left(v_{2}\right)^{2} A(|v|) d v=\frac{1}{35} \int_{\mathbb{R}^{3}}|v|^{6} A(|v|) d v, \\
\int_{\mathbb{R}^{3}}\left(v_{1}\right)^{2}\left(v_{2}\right)^{2}\left(v_{3}\right)^{2} A(|v|) d v=\frac{1}{105} \int_{\mathbb{R}^{3}}|v|^{6} A(|v|) d v .
\end{gathered}
$$

Appendix B: Some details on computations for Constant Cross SECTIONS

In this Appendix, we collect some details about the computations of families of functions $\underline{h}^{W}, \underline{h}^{P}, \underline{h}^{D}, \underline{h}^{Q}$ in case of constant cross sections, the final results being given in Subsections 4.1 - 4.4. We recall that those families of functions allow to construct the viscosity coefficients of Navier-Stokes equations computed in Subsection 4.5.

B.1. Details on computations for $\underline{h}^{W}$. We present here the computations to solve the problem $\mathcal{K}\left(\underline{h}^{W}\right)=\underline{k}^{W}$, with $k^{(i), W}=s^{(i)} \cdot V$, presented in Subsection 4.1.

We begin by computing for all $i, j=1, \ldots, A+B$ the quantity

$$
K_{i j}\left(v \mapsto W_{1}^{(i)} m_{i} v_{1}, v \mapsto W_{1}^{(j)} m_{j} v_{1}\right),
$$

where $W_{1}^{(i)}, W_{1}^{(j)} \in \mathbb{R}$ are constants.

For $i=1, \ldots, A, j=1, \ldots, A$,

$$
\begin{aligned}
& K_{i j}(v\left.\mapsto W_{1}^{(i)} m_{i} v_{1}, v \mapsto W_{1}^{(j)} m_{j} v_{1}\right)(v)=\int_{\mathbb{R}^{3}} \int_{S^{2}} \frac{e^{-\frac{m_{j}}{2}\left|v_{*}\right|^{2}}}{\left(2 \pi / m_{j}\right)^{3 / 2}} \\
& \times\left[W_{1}^{(j)} m_{j}\left(\frac{m_{i} v_{1}+m_{j} v_{1 *}}{m_{i}+m_{j}}-\frac{m_{i}}{m_{i}+m_{j}}\left|v-v_{*}\right| \sigma_{1}\right)\right.
\end{aligned}
$$




$$
\begin{gathered}
+W_{1}^{(i)} m_{i}\left(\frac{m_{i} v_{1}+m_{j} v_{1 *}}{m_{i}+m_{j}}+\frac{m_{j}}{m_{i}+m_{j}}\left|v-v_{*}\right| \sigma_{1}\right) \\
\left.-m_{j} W_{1}^{(j)} v_{1 *}-m_{i} W_{1}^{(i)} v_{1}\right] B_{i j} d \sigma d v_{*} \\
=B_{i j} \mu_{i j}\left(W_{1}^{(j)}-W_{1}^{(i)}\right) v_{1} .
\end{gathered}
$$

For $i=1, \ldots, A, j=A+1, \ldots, A+B$,

$$
\begin{gathered}
K_{i j}\left(v \mapsto W_{1}^{(i)} m_{i} v_{1}, v \mapsto W_{1}^{(j)} m_{j} v_{1}\right)(v)=\int_{\mathbb{R}^{3}} \int_{0}^{\infty} \int_{S^{2}} \int_{0}^{1} \frac{e^{-\frac{m_{j}}{2}\left|v_{*}\right|^{2}-I_{*}}}{\left(2 \pi / m_{j}\right)^{3 / 2}} \\
\times\left[m_{j} W_{1}^{(j)}\left(\frac{m_{i} v_{1}+m_{j} v_{1 *}}{m_{i}+m_{j}}-\frac{m_{i}}{m_{i}+m_{j}} \sqrt{\frac{2 R E}{\mu_{i j}}} \sigma_{1}\right)\right. \\
+m_{i} W_{1}^{(i)}\left(\frac{m_{i} v_{1}+m_{j} v_{1 *}}{m_{i}+m_{j}}+\frac{m_{j}}{m_{i}+m_{j}} \sqrt{\frac{2 R E}{\mu_{i j}}} \sigma_{1}\right) \\
\left.-m_{j} W_{1}^{(j)} v_{1 *}-m_{i} W_{1}^{(i)} v_{1}\right] R^{1 / 2} d R d \sigma d I_{*} d v_{*} \\
=\frac{2}{3} B_{i j} \mu_{i j}\left(W_{1}^{(j)}-W_{1}^{(i)}\right) v_{1} .
\end{gathered}
$$

For $i=A+1, \ldots, A+B, j=1, \ldots, A$,

$$
\begin{gathered}
K_{i j}\left(v \mapsto W_{1}^{(i)} m_{i} v_{1}, v \mapsto W_{1}^{(j)} m_{j} v_{1}\right)(v, I)=\int_{\mathbb{R}^{3}} \int_{S^{2}} \int_{0}^{1} \frac{e^{-\frac{m_{j}}{2}\left|v_{*}\right|^{2}}}{\left(2 \pi / m_{j}\right)^{3 / 2}} \\
\times\left[m_{j} W_{1}^{(j)}\left(\frac{m_{i} v_{1}+m_{j} v_{1 *}}{m_{i}+m_{j}}-\frac{m_{i}}{m_{i}+m_{j}} \sqrt{\frac{2 R E}{\mu_{i j}}} \sigma_{1}\right)\right. \\
+m_{i} W_{1}^{(i)}\left(\frac{m_{i} v_{1}+m_{j} v_{1 *}}{m_{i}+m_{j}}+\frac{m_{j}}{m_{i}+m_{j}} \sqrt{\frac{2 R E}{\mu_{i j}}} \sigma_{1}\right) \\
\left.-m_{j} W_{1}^{(j)} v_{1 *}-m_{i} W_{1}^{(i)} v_{1}\right] B_{i j} R^{1 / 2} d R d \sigma d v_{*} \\
=\frac{2}{3} B_{i j} \mu_{i j}\left(W_{1}^{(j)}-W_{1}^{(i)}\right) v_{1} .
\end{gathered}
$$

For $i=A+1, \ldots, A+B, j=A+1, \ldots, A+B$,

$$
\begin{gathered}
K_{i j}\left(v \mapsto W_{1}^{(i)} m_{i} v_{1}, v \mapsto W_{1}^{(j)} m_{j} v_{1}\right)(v, I)=\int_{\mathbb{R}^{3}} \int_{0}^{\infty} \int_{S^{2}} \int_{0}^{1} \int_{0}^{1} \frac{e^{-\frac{m_{j}}{2}\left|v_{*}\right|^{2}-I_{*}}}{\left(2 \pi / m_{j}\right)^{3 / 2}} \\
\times\left[m_{j} W_{1}^{(j)}\left(\frac{m_{i} v_{1}+m_{j} v_{1 *}}{m_{i}+m_{j}}-\frac{m_{i}}{m_{i}+m_{j}} \sqrt{\frac{2 R E}{\mu_{i j}}} \sigma_{1}\right)\right. \\
\quad+m_{i} W_{1}^{(i)}\left(\frac{m_{i} v_{1}+m_{j} v_{1 *}}{m_{i}+m_{j}}+\frac{m_{j}}{m_{i}+m_{j}} \sqrt{\frac{2 R E}{\mu_{i j}}} \sigma_{1}\right) \\
\left.-m_{j} W_{1}^{(j)} v_{1 *}-m_{i} W_{1}^{(i)} v_{1}\right] B_{i j}(1-R) R^{1 / 2} d R d r d \sigma d I_{*} d v_{*}
\end{gathered}
$$




$$
=\frac{4}{15} B_{i j} \mu_{i j}\left(W_{1}^{(j)}-W_{1}^{(i)}\right) v_{1}
$$

B.2. Details on computations for $\underline{h}^{P}$. We present here the computations concerning the problem $\mathcal{K}\left(\underline{h}^{P}\right)=\underline{k}^{P}$ (see Subsection 4.2).

We consider only the component $p=1, q=2$, the other ones being obtained thanks to the isotropy properties.

For $i=A+1, \ldots, A+B, j=A+1, \ldots, A+B$, and $\Pi_{12}^{(i)}, \Pi_{12}^{(j)}$ real constants,

$$
\begin{gathered}
K_{i j}\left(v \mapsto m_{i} \Pi_{12}^{(i)} v_{1} v_{2}, v \mapsto m_{j} \Pi_{12}^{(j)} v_{1} v_{2}\right)(v, I)=\int_{\mathbb{R}^{3}} \int_{0}^{\infty} \int_{S^{2}} \int_{0}^{1} \int_{0}^{1} \frac{e^{-\frac{m_{j}}{2}\left|v_{*}\right|^{2}-I_{*}}}{\left(2 \pi / m_{j}\right)^{3 / 2}} \\
\times\left[m_{j} \Pi_{12}^{(j)}\left(\frac{m_{i} v_{1}+m_{j} v_{1 *}}{m_{i}+m_{j}}-\frac{m_{i}}{m_{i}+m_{j}} \sqrt{\frac{2 R E}{\mu_{i j}}} \sigma_{1}\right)\right. \\
\times\left(\frac{m_{i} v_{2}+m_{j} v_{2 *}}{m_{i}+m_{j}}-\frac{m_{i}}{m_{i}+m_{j}} \sqrt{\frac{2 R E}{\mu_{i j}}} \sigma_{2}\right) \\
+m_{i} \Pi_{12}^{(i)}\left(\frac{m_{i} v_{1}+m_{j} v_{1 *}}{m_{i}+m_{j}}+\frac{m_{j}}{m_{i}+m_{j}} \sqrt{\frac{2 R E}{\mu_{i j}}} \sigma_{1}\right) \\
\times\left(\frac{m_{i} v_{2}+m_{j} v_{2 *}}{m_{i}+m_{j}}+\frac{m_{j}}{m_{i}+m_{j}} \sqrt{\left.\frac{2 R E}{\mu_{i j}} \sigma_{2}\right)}\right. \\
\left.-m_{j} \Pi_{12}^{(j)} v_{1 *} v_{2 *}-m_{i} \Pi_{12}^{(i)} v_{1} v_{2}\right] B_{i j}(1-R) R^{1 / 2} d R d r d \sigma d I_{*} d v_{*} \\
=\frac{4}{15} B_{i j} \mu_{i j}^{2}\left(\frac{\Pi_{12}^{(j)}-2 \Pi_{12}^{(i)}}{m_{j}}-\frac{\Pi_{12}^{(i)}}{m_{i}}\right) v_{1} v_{2} .
\end{gathered}
$$

In the same way, for $i=1, \ldots, A, j=1, \ldots, A$,

$K_{i j}\left(v \mapsto m_{i} \Pi_{12}^{(i)} v_{1} v_{2}, v \mapsto m_{j} \Pi_{12}^{(j)} v_{1} v_{2}\right)(v)=B_{i j} \mu_{i j}^{2}\left(\frac{\Pi_{12}^{(j)}-2 \Pi_{12}^{(i)}}{m_{j}}-\frac{\Pi_{12}^{(i)}}{m_{i}}\right) v_{1} v_{2}$,

for $i=1, \ldots, A, j=A+1, \ldots, A+B$,

$K_{i j}\left(v \mapsto m_{i} \Pi_{12}^{(i)} v_{1} v_{2}, v \mapsto m_{j} \Pi_{12}^{(j)} v_{1} v_{2}\right)(v)=\frac{2}{3} B_{i j} \mu_{i j}^{2}\left(\frac{\Pi_{12}^{(j)}-2 \Pi_{12}^{(i)}}{m_{j}}-\frac{\Pi_{12}^{(i)}}{m_{i}}\right) v_{1} v_{2}$,

for $i=A+1, \ldots, A+B, j=1, \ldots, A$,

$K_{i j}\left(v \mapsto m_{i} \Pi_{12}^{(i)} v_{1} v_{2}, v \mapsto m_{j} \Pi_{12}^{(j)} v_{1} v_{2}\right)(v, I)=\frac{2}{3} B_{i j} \mu_{i j}^{2}\left(\frac{\Pi_{12}^{(j)}-2 \Pi_{12}^{(i)}}{m_{j}}-\frac{\Pi_{12}^{(i)}}{m_{i}}\right) v_{1} v_{2}$.

Finally, with the notations of Subsection 4.1 for $\tilde{B}_{i j}$, for $i=1, \ldots, A+B, j=$ $1, \ldots, A+B$

$$
K_{i j}\left(v \mapsto m_{i} \Pi_{12}^{(i)} v_{1} v_{2}, v \mapsto m_{j} \Pi_{12}^{(j)} v_{1} v_{2}\right)=\tilde{B}_{i j} \mu_{i j}^{2}\left(\frac{\Pi_{12}^{(j)}-2 \Pi_{12}^{(i)}}{m_{j}}-\frac{\Pi_{12}^{(i)}}{m_{i}}\right) v_{1} v_{2} .
$$


B.3. Details on computations for $\underline{h}^{D}$. We present here the computations concerning the problem $\mathcal{K}\left(\underline{h}^{D}\right)=\underline{k}^{D}$ (see Subsection 4.3). We skip a lot of intermediate steps, that may be recovered using the integrals reported in Appendix A, analogously to previous paragraphs.

We introduce indeterminate constant coefficients $\Delta^{(i)}$ for $i=1, \ldots, A+B$ and $\widetilde{\Delta}^{(i)}$ for $i=A+1, \ldots, A+B$.

- $\quad$ For $i=1, \ldots, A$ and $j=1, \ldots, A$, we get

$$
\begin{gathered}
B_{i j}^{-1} K_{i j}\left(v \mapsto \Delta^{(i)}\left(m_{i}|v|^{2}-3\right), \quad v \mapsto \Delta^{(j)}\left(m_{j}|v|^{2}-3\right)\right)(v)= \\
=\int_{\mathbb{R}^{3}} \int_{S^{2}}\left(\frac{m_{j}}{2 \pi}\right)^{3 / 2} e^{-\frac{1}{2} m_{j}\left|v_{*}\right|^{2}}\left[\Delta^{(j)}\left\{m_{j}\left|\frac{m_{i} v+m_{j} v_{*}}{m_{i}+m_{j}}-\frac{m_{i}}{m_{i}+m_{j}}\right| v-v_{*}|\sigma|^{2}-3\right\}\right. \\
+\Delta^{(i)}\left\{m_{i}\left|\frac{m_{i} v+m_{j} v_{*}}{m_{i}+m_{j}}+\frac{m_{j}}{m_{i}+m_{j}}\right| v-v_{*}|\sigma|^{2}-3\right\}-\Delta^{(j)}\left(m_{j}\left|v_{*}\right|^{2}-3\right) \\
\left.-\Delta^{(i)}\left(m_{i}|v|^{2}-3\right)\right] d \sigma d v_{*}=\frac{2 \mu_{i j}}{m_{i}+m_{j}}\left(\Delta^{(j)}-\Delta^{(i)}\right)\left(m_{i}|v|^{2}-3\right) .
\end{gathered}
$$

- $\quad$ For $i=1, \ldots, A$ and $j=A+1, \ldots, A+B$, we get

$$
\begin{gathered}
B_{i j}^{-1} K_{i j}\left(v \mapsto \Delta^{(i)}\left(m_{i}|v|^{2}-3\right), \quad(v, I) \mapsto \Delta^{(j)}\left(m_{j}|v|^{2}-3\right)+\widetilde{\Delta}^{(j)}(I-1)\right)(v)= \\
=\int_{\mathbb{R}^{3}} \int_{S^{2}} \int_{0}^{\infty} \int_{0}^{1}\left(\frac{m_{j}}{2 \pi}\right)^{3 / 2} e^{-\frac{1}{2} m_{j}\left|v_{*}\right|^{2}-I_{*}}\left[\Delta^{(j)}\left\{m_{j}\left|\frac{m_{i} v+m_{j} v_{*}}{m_{i}+m_{j}}-\frac{m_{i}}{m_{i}+m_{j}} \sqrt{\frac{2 R E}{\mu_{i j}}} \sigma\right|^{2}-3\right\}\right. \\
+\widetilde{\Delta}^{(j)}((1-R) E-1)+\Delta^{(i)}\left\{m_{i}\left|\frac{m_{i} v+m_{j} v_{*}}{m_{i}+m_{j}}+\frac{m_{j}}{m_{i}+m_{j}} \sqrt{\frac{2 R E}{\mu_{i j}}} \sigma\right|^{2}-3\right\} \\
\left.-\Delta^{(j)}\left(m_{j}\left|v_{*}\right|^{2}-3\right)-\widetilde{\Delta}^{(j)}\left(I_{*}-1\right)-\Delta^{(i)}\left(m_{i}|v|^{2}-3\right)\right] \sqrt{R} d R d I_{*} d \sigma d v_{*} \\
=\frac{2}{15} \frac{m_{j}}{m_{i}+m_{j}}\left(\Delta^{(j)} \frac{8 m_{i}}{m_{i}+m_{j}}+\widetilde{\Delta}^{(j)}\right)\left(m_{i}|v|^{2}-3\right) \\
+\Delta^{(i)}\left[-\frac{4}{3} \frac{m_{j}}{\left(m_{i}+m_{j}\right)^{2}}\left(m_{i}+\frac{1}{5} m_{j}\right)\right]\left(m_{i}|v|^{2}-3\right) .
\end{gathered}
$$

- $\quad$ For $i=A+1, \ldots, A+B$ and $j=1, \ldots, A$, we get

$$
\begin{aligned}
& B_{i j}^{-1} K_{i j}\left((v, I) \mapsto \Delta^{(i)}\left(m_{i}|v|^{2}-3\right)+\widetilde{\Delta}^{(i)}(I-1), \quad v \mapsto \Delta^{(j)}\left(m_{j}|v|^{2}-3\right)\right)(v, I)= \\
& =\int_{\mathbb{R}^{3}} \int_{S^{2}} \int_{0}^{1}\left(\frac{m_{j}}{2 \pi}\right)^{3 / 2} e^{-\frac{1}{2} m_{j}\left|v_{*}\right|^{2}}\left[\Delta^{(j)}\left\{m_{j}\left|\frac{m_{i} v+m_{j} v_{*}}{m_{i}+m_{j}}-\frac{m_{i}}{m_{i}+m_{j}} \sqrt{\frac{2 R E}{\mu_{i j}}} \sigma\right|^{2}-3\right\}\right. \\
& +\Delta^{(i)}\left\{m_{i}\left|\frac{m_{i} v+m_{j} v_{*}}{m_{i}+m_{j}}+\frac{m_{j}}{m_{i}+m_{j}} \sqrt{\frac{2 R E}{\mu_{i j}}} \sigma\right|^{2}-3\right\}+\widetilde{\Delta}^{(i)}[(1-R) E-1] \\
& \left.-\Delta^{(j)}\left(m_{j}\left|v_{*}\right|^{2}-3\right)-\Delta^{(i)} m_{i}\left(|v|^{2}-3\right)-\widetilde{\Delta}^{(i)}(I-1)\right] \sqrt{R} d R d \sigma d v_{*}
\end{aligned}
$$




$$
\begin{gathered}
=\Delta^{(j)}\left[\frac{16}{15} \frac{\mu_{i j}}{m_{i}+m_{j}}\left(m_{i}|v|^{2}-3\right)+\frac{4}{5} \frac{m_{i}}{m_{i}+m_{j}}(I-1)\right] \\
-\frac{2}{15} \frac{m_{j}}{m_{i}+m_{j}}\left(2 \Delta^{(i)} \frac{5 m_{i}+m_{j}}{m_{i}+m_{j}}-\widetilde{\Delta}^{(i)}\right)\left(m_{i}|v|^{2}-3\right)+\frac{2}{5}\left(\Delta^{(i)} \frac{2 m_{j}}{m_{i}+m_{j}}-\widetilde{\Delta}^{(i)}\right)(I-1) .
\end{gathered}
$$

- For $i=A+1, \ldots, A+B$ and $j=A+1, \ldots, A+B$, we get

$$
\begin{gathered}
B_{i j}^{-1} K_{i j}\left((v, I) \mapsto \Delta^{(i)}\left(m_{i}|v|^{2}-3\right)+\widetilde{\Delta}^{(i)}(I-1), \quad(v, I) \mapsto \Delta^{(j)}\left(m_{j}|v|^{2}-3\right)+\widetilde{\Delta}^{(j)}(I-1)\right)(v, I)= \\
=\int_{\mathbb{R}^{3}} \int_{S^{2}} \int_{0}^{\infty} \int_{0}^{1} \int_{0}^{1}\left(\frac{m_{j}}{2 \pi}\right)^{3 / 2} e^{-\frac{1}{2} m_{j}\left|v_{*}\right|^{2}-I_{*}}\left[\Delta ^ { ( j ) } \left\{m_{j} \mid \frac{m_{i} v+m_{j} v_{*}}{m_{i}+m_{j}}-\frac{m_{i}}{m_{i}+m_{j}} \sqrt{\left.\frac{2 R E}{\mu_{i j}} \sigma\right|^{2}}\right.\right. \\
-3\}+\widetilde{\Delta}^{(j)}[(1-r)(1-R) E-1]+\Delta^{(i)}\left\{m_{i}\left|\frac{m_{i} v+m_{j} v_{*}}{m_{i}+m_{j}}+\frac{m_{j}}{m_{i}+m_{j}} \sqrt{\frac{2 R E}{\mu_{i j}}} \sigma\right|^{2}-3\right\} \\
\left.+\widetilde{\Delta}^{(i)}[r(1-R) E-1]-\Delta^{(j)}\left(m_{j}\left|v_{*}\right|^{2}-3\right)-\widetilde{\Delta}^{(j)}\left(I_{*}-1\right)-\Delta^{(i)} m_{i}\left(|v|^{2}-3\right)-\widetilde{\Delta}^{(i)}(I-1)\right] \\
\times(1-R) \sqrt{R} d R d r d I_{*} d \sigma d v_{*} \\
=\frac{4}{21} \frac{m_{j}}{\left(m_{i}+m_{j}\right)^{2}}\left(2 \Delta^{(j)} m_{i}+\frac{1}{5} \widetilde{\Delta}^{(j)}\left(m_{i}+m_{j}\right)\right)\left(m_{i}|v|^{2}-3\right) \\
+\frac{8}{35}\left(\Delta^{(j)} \frac{m_{i}}{m_{i}+m_{j}}+\frac{1}{3} \widetilde{\Delta}^{(j)}\right)(I-1) \\
-\frac{4}{15} \frac{m_{j}}{\left(m_{i}+m_{j}\right)^{2}}\left[\Delta^{(i)}\left(2 m_{i}+\frac{4}{7} m_{j}\right)-\frac{1}{7} \widetilde{\Delta}^{(i)}\left(m_{i}+m_{j}\right)\right]\left(m_{i}|v|^{2}-3\right) \\
+\frac{4}{7}\left(\frac{2}{5} \Delta^{(i)} \frac{m_{j}}{m_{i}+m_{j}}-\frac{1}{3} \widetilde{\Delta}^{(i)}\right)(I-1) .
\end{gathered}
$$

B.4. Details on computations for $\underline{h}^{Q}$. We present here the computations concerning the problem $\mathcal{K}\left(\underline{h}^{Q}\right)=\underline{k}^{Q}$ (see Subsection 4.4).

We test for that the effect of $K_{i j}$ on combinations of $|v|^{2} v_{1}$ and $v_{1}$.

We introduce indeterminate constant coefficients $Q^{(i)}$ for $i=1, \ldots, A+B$ and $\tilde{Q}^{(i)}$ for $i=A+1, \ldots, A+B$.

- $\quad$ For $i=1, \ldots, A$ and $j=1, \ldots, A$, we get

$$
\begin{gathered}
B_{i j}^{-1} K_{i j}\left(v \mapsto Q^{(i)} m_{i}|v|^{2} v_{1}, \quad v \mapsto Q^{(j)} m_{j}|v|^{2} v_{1}\right)(v)= \\
=\int_{\mathbb{R}^{3}} \int_{S^{2}}\left(\frac{m_{j}}{2 \pi}\right)^{3 / 2} e^{-\frac{1}{2} m_{j}\left|v_{*}\right|^{2}}\left[Q^{(j)} m_{j}\left|\frac{m_{i} v+m_{j} v_{*}}{m_{i}+m_{j}}-\frac{m_{i}}{m_{i}+m_{j}}\right| v-v_{*}|\sigma|^{2}\right. \\
\times\left(\frac{m_{i} v_{1}+m_{j} v_{1 *}}{m_{i}+m_{j}}-\frac{m_{i}}{m_{i}+m_{j}}\left|v-v_{*}\right| \sigma_{1}\right)+Q^{(i)} m_{i}\left|\frac{m_{i} v+m_{j} v_{*}}{m_{i}+m_{j}}+\frac{m_{j}}{m_{i}+m_{j}}\right| v-v_{*}|\sigma|^{2} \\
\left.\times\left(\frac{m_{i} v_{1}+m_{j} v_{1 *}}{m_{i}+m_{j}}+\frac{m_{j}}{m_{i}+m_{j}}\left|v-v_{*}\right| \sigma_{1}\right)-Q^{(j)} m_{j}\left|v_{*}\right|^{2} v_{1 *}-Q^{(i)} m_{i}|v|^{2} v_{1}\right] d \sigma d v_{*} \\
=Q^{(j)} \frac{m_{i}}{\left(m_{i}+m_{j}\right)^{3}}\left[\frac{8}{3} m_{i}^{2} m_{j}|v|^{2} v_{1}+5\left(m_{i}^{2}+m_{j}^{2}-\frac{2}{3} m_{i} m_{j}\right) v_{1}\right] \\
+Q^{(i)} \frac{m_{i}}{\left(m_{i}+m_{j}\right)^{3}}\left[-m_{j}\left(3 m_{i}^{2}+m_{j}^{2}+\frac{4}{3} m_{i} m_{j}\right)|v|^{2} v_{1}+10 m_{j}\left(m_{i}-\frac{1}{3} m_{j}\right) v_{1}\right] .
\end{gathered}
$$


- $\quad$ For $i=1, \ldots, A$ and $j=A+1, \ldots, A+B$, we get

$$
\begin{aligned}
& B_{i j}^{-1} K_{i j}\left(v \mapsto Q^{(i)} m_{i}|v|^{2} v_{1}, \quad(v, I) \mapsto Q^{(j)} m_{j}|v|^{2} v_{1}+\widetilde{Q}^{(j)} I v_{1}\right)(v)= \\
& =\int_{\mathbb{R}^{3}} \int_{S^{2}} \int_{0}^{\infty} \int_{0}^{1}\left(\frac{m_{j}}{2 \pi}\right)^{3 / 2} e^{-\frac{1}{2} m_{j}\left|v_{*}\right|^{2}-I_{*}}\left[Q^{(j)} m_{j}\left|\frac{m_{i} v+m_{j} v_{*}}{m_{i}+m_{j}}-\frac{m_{i}}{m_{i}+m_{j}} \sqrt{\frac{2 R E}{\mu_{i j}}} \sigma\right|^{2}\right. \\
& \times\left(\frac{m_{i} v_{1}+m_{j} v_{1 *}}{m_{i}+m_{j}}-\frac{m_{i}}{m_{i}+m_{j}} \sqrt{\frac{2 R E}{\mu_{i j}}} \sigma_{1}\right)+\widetilde{Q}^{(j)}(1-R) E\left(\frac{m_{i} v_{1}+m_{j} v_{1 *}}{m_{i}+m_{j}}-\frac{m_{i}}{m_{i}+m_{j}} \sqrt{\frac{2 R E}{\mu_{i j}}} \sigma_{1}\right) \\
& +Q^{(i)} m_{i}\left|\frac{m_{i} v+m_{j} v_{*}}{m_{i}+m_{j}}+\frac{m_{j}}{m_{i}+m_{j}} \sqrt{\frac{2 R E}{\mu_{i j}}} \sigma\right|^{2}\left(\frac{m_{i} v_{1}+m_{j} v_{1 *}}{m_{i}+m_{j}}+\frac{m_{j}}{m_{i}+m_{j}} \sqrt{\frac{2 R E}{\mu_{i j}}} \sigma_{1}\right) \\
& \left.=Q^{(j)} m_{j}\left|v_{*}\right|^{2} v_{1 *}-\widetilde{Q}^{(j)} I_{*} v_{1 *}-Q^{(i)} m_{i}|v|^{2} v_{1}\right] \sqrt{R} d R d I_{*} d \sigma d v_{*} \\
& \frac{m_{i}}{\left(m_{i}+m_{j}\right)^{3}}\left[2 m_{i}^{2} m_{j}|v|^{2} v_{1}+5\left(m_{i}^{2}+m_{j}^{2}\right) v_{1}\right]+\widetilde{Q}^{(j)} \frac{2}{3} \frac{m_{i}^{2}}{\left(m_{i}+m_{j}\right)^{2}}\left[\frac{1}{5} m_{j}|v|^{2} v_{1}+v_{1}\right] \\
& +Q^{(i)} \frac{2}{3} \frac{m_{i} m_{j}}{\left(m_{i}+m_{j}\right)^{3}}\left[-\left(3 m_{i}^{2}+2 m_{i} m_{j}+m_{j}^{2}\right)|v|^{2} v_{1}+10 m_{i} v_{1}\right] .
\end{aligned}
$$

- $\quad$ For $i=A+1, \ldots, A+B$ and $j=1, \ldots, A$, we get

$$
\begin{aligned}
& B_{i j}^{-1} K_{i j}\left((v, I) \mapsto Q^{(i)} m_{i}|v|^{2} v_{1}+\widetilde{Q}^{(i)} I v_{1}, \quad v \mapsto Q^{(j)} m_{j}|v|^{2} v_{1}\right)(v, I)= \\
= & \int_{\mathbb{R}^{3}} \int_{S^{2}} \int_{0}^{1}\left(\frac{m_{j}}{2 \pi}\right)^{3 / 2} e^{-\frac{1}{2} m_{j}\left|v_{*}\right|^{2}}\left[Q^{(j)} m_{j}\left|\frac{m_{i} v+m_{j} v_{*}}{m_{i}+m_{j}}-\frac{m_{i}}{m_{i}+m_{j}} \sqrt{\frac{2 R E}{\mu_{i j}}} \sigma\right|^{2}\right. \\
\times & \left(\frac{m_{i} v_{1}+m_{j} v_{1 *}}{m_{i}+m_{j}}-\frac{m_{i}}{m_{i}+m_{j}} \sqrt{\frac{2 R E}{\mu_{i j}}} \sigma_{1}\right)+Q^{(i)} m_{i}\left|\frac{m_{i} v+m_{j} v_{*}}{m_{i}+m_{j}}+\frac{m_{j}}{m_{i}+m_{j}} \sqrt{\frac{2 R E}{\mu_{i j}}} \sigma\right|^{2} \\
& \times\left(\frac{m_{i} v_{1}+m_{j} v_{1 *}}{m_{i}+m_{j}}+\frac{m_{j}}{m_{i}+m_{j}} \sqrt{\frac{2 R E}{\mu_{i j}}} \sigma_{1}\right)+\widetilde{Q}^{(i)}(1-R) E\left(\frac{m_{i} v_{1}+m_{j} v_{1 *}}{m_{i}+m_{j}}\right. \\
+ & \left.\left.\frac{m_{j}}{m_{i}+m_{j}} \sqrt{\frac{2 R E}{\mu_{i j}}} \sigma_{1}\right)-Q^{(j)} m_{j}\left|v_{*}\right|^{2} v_{1 *}-Q^{(i)} m_{i}|v|^{2} v_{1}-\widetilde{Q}^{(i)} I v_{1}\right] \sqrt{R} d R d \sigma d v_{*} \\
= & Q^{(j)} \frac{m_{i}}{\left(m_{i}+m_{j}\right)^{3}}\left[\frac{4}{3} m_{i}^{2} m_{j}|v|^{2} v_{1}+\frac{4}{3} m_{i}\left(m_{i}+m_{j}\right) I v_{1}+2\left(m_{i}^{2}-\frac{2}{3} m_{i} m_{j}+\frac{5}{3} m_{j}^{2}\right) v_{1}\right] \\
+ & Q^{(i)} \frac{m_{i} m_{j}}{\left(m_{i}+m_{j}\right)^{3}}\left[-\left(2 m_{i}^{2}+\frac{4}{3} m_{i} m_{j}+\frac{2}{3} m_{j}^{2}\right)|v|^{2} v_{1}+\frac{4}{3}\left(m_{i}+m_{j}\right) I v_{1}+\frac{4}{3}\left(4 m_{i}-m_{j}\right) v_{1}\right] \\
+ & \widetilde{Q}^{(i)} \frac{2}{15} \frac{1}{\left(m_{i}+m_{j}\right)^{2}}\left[m_{i}^{2} m_{j}|v|^{2} v_{1}-\left(3 m_{i}^{2}+8 m_{i} m_{j}+5 m_{j}^{2}\right) I v_{1}+\left(3 m_{i}-2 m_{j}\right) m_{i} v_{1}\right] .
\end{aligned}
$$

- $\quad$ For $i=A+1, \ldots, A+B$ and $j=A+1, \ldots, A+B$, we get

$$
B_{i j}^{-1} K_{i j}\left((v, I) \mapsto Q^{(i)} m_{i}|v|^{2} v_{1}+\widetilde{Q}^{(i)} I v_{1}, \quad(v, I) \mapsto Q^{(j)} m_{j}|v|^{2} v_{1}+\widetilde{Q}^{(j)} I v_{1}\right)(v, I)=
$$




$$
\begin{aligned}
& =\int_{\mathbb{R}^{3}} \int_{S^{2}} \int_{0}^{\infty} \int_{0}^{1} \int_{0}^{1}\left(\frac{m_{j}}{2 \pi}\right)^{3 / 2} e^{-\frac{1}{2} m_{j}\left|v_{*}\right|^{2}-I_{*}}\left[\left(Q^{(j)} m_{j}\left|\frac{m_{i} v+m_{j} v_{*}}{m_{i}+m_{j}}-\frac{m_{i}}{m_{i}+m_{j}} \sqrt{\frac{2 R E}{\mu_{i j}}} \sigma\right|^{2}\right.\right. \\
& \left.+\widetilde{Q}^{(j)}(1-r)(1-R) E\right)\left(\frac{m_{i} v_{1}+m_{j} v_{1 *}}{m_{i}+m_{j}}-\frac{m_{i}}{m_{i}+m_{j}} \sqrt{\frac{2 R E}{\mu_{i j}}} \sigma_{1}\right) \\
& +\left(Q^{(i)} m_{i}\left|\frac{m_{i} v+m_{j} v_{*}}{m_{i}+m_{j}}+\frac{m_{j}}{m_{i}+m_{j}} \sqrt{\frac{2 R E}{\mu_{i j}}} \sigma\right|^{2}+\widetilde{Q}^{(i)} r(1-R) E\right) \\
& \times\left(\frac{m_{i} v_{1}+m_{j} v_{1 *}}{m_{i}+m_{j}}+\frac{m_{j}}{m_{i}+m_{j}} \sqrt{\frac{2 R E}{\mu_{i j}}} \sigma_{1}\right) \\
& \left.-Q^{(j)} m_{j}\left|v_{*}\right|^{2} v_{1 *}-\widetilde{Q}^{(j)} I_{*} v_{1 *}-Q^{(i)} m_{i}|v|^{2} v_{1}-\widetilde{Q}^{(i)} I v_{1}\right](1-R) \sqrt{R} d R d r d I_{*} d \sigma d v_{*} \\
& =Q^{(j)} \frac{m_{i}}{\left(m_{i}+m_{j}\right)^{3}}\left[\frac{16}{35} m_{i}^{2} m_{j}|v|^{2} v_{1}+\frac{8}{21} m_{i}\left(m_{i}+m_{j}\right) I v_{1}+\left(\frac{20}{21} m_{i}^{2}+\frac{4}{3} m_{j}^{2}\right) v_{1}\right] \\
& +\widetilde{Q}^{(j)} \frac{8}{105} \frac{m_{i}}{\left(m_{i}+m_{j}\right)^{2}}\left[\frac{1}{2} m_{i} m_{j}|v|^{2} v_{1}+\left(m_{i}+m_{j}\right) I v_{1}+\frac{5}{2} m_{i} v_{1}\right] \\
& +Q^{(i)} \frac{m_{i}}{\left(m_{i}+m_{j}\right)^{3}}\left[-\frac{4}{5} m_{j}\left(m_{i}^{2}+\frac{16}{21} m_{i} m_{j}+\frac{1}{3} m_{j}^{2}\right)|v|^{2} v_{1}+\frac{8}{21} m_{j}\left(m_{i}+m_{j}\right) I v_{1}+\frac{16}{7} m_{i} m_{j} v_{1}\right] \\
& +\widetilde{Q}^{(i)} \frac{8}{105} \frac{1}{\left(m_{i}+m_{j}\right)^{2}}\left[\frac{1}{2} m_{i}^{2} m_{j}|v|^{2} v_{1}-\frac{1}{2}\left(5 m_{i}+7 m_{j}\right)\left(m_{i}+m_{j}\right) I v_{1}+\frac{5}{2} m_{i}^{2} v_{1}\right] .
\end{aligned}
$$

\section{REFERENCES}

[1] P. Andries, P. Le Tallec, J.P. Perlat, B. Perthame Entropy condition for the ES BGK model of Boltzmann equation for mono and polyatomic gases. Eur. J. Mech. B/fluids, 19, 813-830 (2000).

[2] C. Bardos, Une interprétation des relations existant entre les équations de Boltzmann, de Navier-Stokes et d'Euler à l'aide de l'entropie, Math. Aplic. Comp., 6, n. 1,(1987), 97-117.

[3] M. Bisi, M.J. Cáceres, A BGK relaxation model for polyatomic gas mixtures, Commun. Math. Sci. 14 (2016), 297-325.

[4] M. Bisi, M. Groppi, G. Spiga, Kinetic Bhatnagar-Gross-Krook model for fast reactive mixtures and its hydrodynamic limit, Phys. Rev. E 81 (2010), 036327 (pp. 1-9).

[5] M. Bisi, T. Ruggeri, G. Spiga, Dynamical pressure in a polyatomic gas: interplay between kinetic theory and Extended Thermodynamics, Kinet. Relat. Models, 11 (2018), 71-95.

[6] C. Borgnakke, P.S. Larsen, Statistical collision model for Monte-Carlo simulation of polyatomic mixtures, Journ. Comput. Phys., 18, 405-420, (1975)

[7] L. Boudin, B. Grec, M. Pavic, and F. Salvarani, Diffusion asymptotics of a kinetic model for gaseous mixtures, Kinet. Relat. Models 6, 1 (2013) 137-157.

[8] J.F. Bourgat, L. Desvillettes, P. Le Tallec and B. Perthame: Microreversible Collisions for Polyatomic Gases and Boltzmann's Theorem, European Journal of Mechanics, B/ Fluids, 13, n.2, (1994), 237-254.

[9] M. Briant, E. Daus The Boltzmann equation for a multi-species mixture close to global equilibrium, Archive Rational Mech. Anal., 222, n.3, (2016), 1367-1443.

[10] S. Brull, J.Schneider On the Ellipsoidal Statistical Model for polyatomic gases. Cont. Mech. Thermodyn. 20, (2009), no.8, 489-508.

[11] C. Cercignani, The Boltzmann Equation and its Applications, Springer, New York (1988).

[12] E. Daus, A. Jüngel, C. Mouhot, and N. Zamponi Hypocoercivity for a linearized multispecies Boltzmann system. SIAM J. Math. Anal. 48, n.1, (2016), 538-568. 
[13] L. Desvillettes, Convergence to the Thermodynamical Equilibrium, In Trends in Applications of Mathematics to Mechanics, Monographs and Surveys in Pure and Applied Mathematics, Vol. 106, Chapman \& Hall, Boca Raton, (2000), 115-126.

[14] L. Desvillettes, Sur un Modèle de type Borgnakke-Larsen Conduisant à des Lois d'Energie Non-linéaires en Température pour les Gaz Parfaits Polyatomiques, Annales de la Faculté des Sciences de Toulouse, Série 6, 6, n.2, (1997), 257-262.

[15] L. Desvillettes and F. Golse: A Remark Concerning the Chapman-Enskog Asymptotics, in Advances in Kinetic Theory and Computing, Series on Advances in Mathematics for Applied Sciences, Vol. 22, World Scientific Publications, Singapour, (1994), 191-203.

[16] L. Desvillettes, R. Monaco and F. Salvarani: A Kinetic Model Allowing to Obtain the Energy Law of Polytropic Gases in the Presence of Chemical Reactions, European Journal of Mechanics B/Fluids, 24, (2005), 219-236.

[17] B. Dubroca and L. Mieussens. A conservative and entropic discrete-velocity model for rarefied polyatomic gases. In CEMRACS 1999 (Orsay), volume 10 of ESAIM Proc., pages 127-139 (electronic). Soc. Math. Appl. Indust., Paris, 1999.

[18] A. Ern and V. Giovangigli, Multicomponent Transport Algorithms, Lecture Notes in Physics Monographs, M 24, 1994.

[19] A. Ern and V. Giovangigli, The kinetic equilibrium regime, Physica A, 260, 49-72, (1998).

[20] H. Funagane, S.Takata, K.Aoki, K.Kugimoto, Poiseuille flow and thermal transpiration of a rarefied polyatomic gas through a circular tube with applications to microflows, Boll. Unione Mat. Ital. (9) 4 (2011), no. 1, 19-46.

[21] V. Giovangigli Multicomponent flow modeling, MESST Series, Birkhauser Boston, 1999.

[22] F. Golse, Fluid dynamic limits of the kinetic theory of gases, in From Particle Systems to Partial Differential Equations (Particle Systems and PDEs, Braga, Portugal, December 2012), C. Bernardin, P. Goncalves Eds., "Springer Proceedings in Mathematics \& Statistics" (2014), pp. 3-91.

[23] M. Groppi, G. Spiga, Kinetic approach to chemical reactions and inelastic transitions in a rarefied gas, J. Math. Chem., 26 (1999), 197-219.

[24] S. Kawashima, A. Matsumura, T. Nishida, On the fluid dynamical approximation to the Boltzmann equation at the level of the Navier-Stokes equation, Commun. Math. Phys. $\mathbf{7 0}$ (1979), 97-124.

[25] S. Kosuge, K. Aoki and T. Goto, Shock wave structure in polyatomic gases: Numerical analysis using a model Boltzmann equation, AIP Conf.Proc., 1786, 180004, (2016).

[26] P. Le Tallec, A hierarchy of hyperbolic models linking Boltzmann to Navier Stokes equations for polyatomic gases, ZAMM, 80,11-12, 779-790, (2000).

[27] F.R. McCourt, J.J. Beenakker, W.E. Köhler, and I. Kuscer, Non Equilibrium Phenomena in Polyatomic Gases. Volume I: Dilute Gases, Clarendon Press, Oxford (1990).

[28] L. Monchick, K.S. Yun, E.A. Mason, Formal kinetic theory of transport phenomena in polyatomic gas mixtures, J. Chem. Phys., 39 (1963) 654-669.

[29] E. Nagnibeda and E. Kustova, Non-equilibrium reacting gas flow, Springer Verlag, Berlin, (2009).

[30] M. Pavić, T. Ruggeri, S. Simić, Maximum entropy principle for polyatomic gases, Physica A 392 (2013), 1302-1317.

[31] T. Ruggeri, M. Sugiyama, Rational extended thermodynamics beyond the monatomic gas, Springer International Publishing, Switzerland, 2015.

[32] S. Takata, H. Funagane, K.Aoki, Fluid modeling for the Knudsen compressor: case of polyatomic gases, Kinet. Relat. Models 3 (2010), no. 2, 353-372.

[33] L. Waldmann, E. Trübenbacher, Formale kinetische Theorie von Gasgemischen aus anregbaren Molekülen, Zeitschr. Naturforschg., 17a (1962) 363-376.

[34] V. M. Zhdanov, Transport processes in multicomponent plasmas, Taylor and Francis, London, 2002. 
(C.B.) CEA-CESTA, 15 avenue des sablières - CS 6000133116 Le Barp Cedex, France

E-mail address: celine.baranger@cea.fr

(M.B.) University of Parma, Dept. of Mathematics, Physics and Computer Sciences, Parco Area delle Scienze 53/A, I-43124, Parma, Italy.

E-mail address: marzia.bisi@unipr.it

(S.B.) Univ. Bordeaux, CNRS, Bordeaux INP, IMB, UMR 5251, F-33400 Talence, France.

E-mail address: Stephane.Brull@math.u-bordeaux.fr

(L.D.) Univ. Paris Diderot, Sorbonne Paris Cité, Institut de Mathématiques de Jussieu - Paris Rive Gauche, UMR 7586, CNRS, Sorbonne Universités, UPMC Univ. Paris 06, F-75013, Paris, France.

E-mail address: desvillettes@math.univ-paris-diderot.fr 